Article

\title{
Invariant Solutions of the Wave Equation on Static Spherically Symmetric Spacetimes Admitting $G_{7}$ Isometry Algebra
}

\author{
Hassan Azad ${ }^{1}$, Khaleel Anaya ${ }^{2}$, Ahmad Y. Al-Dweik ${ }^{3}$ (D) and M. T. Mustafa ${ }^{3, *}$ \\ 1 Abdus Salam School of Mathematical Sciences, GC University, Lahore 54600, Pakistan; \\ hassan.azad@sms.edu.pk \\ 2 Department of Mathematics and Statistics, King Fahd University of Petroleum and Minerals, \\ Dhahran 31261, Saudi Arabia; khalil.anaya1@gmail.com \\ 3 Department of Mathematics, Statistics and Physics, Qatar University, Doha 2713, Qatar; aydweik@qu.edu.qa \\ * Correspondence: tahir.mustafa@qu.edu.qa
}

Received: 30 October 2018; Accepted: 19 November 2018; Published: 23 November 2018

\begin{abstract}
Algorithms to construct the optimal systems of dimension of at most three of Lie algebras are given. These algorithms are applied to determine the Lie algebra structure and optimal systems of the symmetries of the wave equation on static spherically symmetric spacetimes admitting $G_{7}$ as an isometry algebra. Joint invariants and invariant solutions corresponding to three-dimensional optimal systems are also determined.
\end{abstract}

Keywords: wave equation; spherically symmetric spacetimes; lie symmetries; roots; optimal systems; invariant solutions

\section{Introduction}

It was shown in [1-3] that spherically symmetric spacetimes belong to one of the following four classes according to their isometries and metrics:

- $G_{10}$ corresponding to the static spacetimes Minkowski, de Sitter and anti de Sitter.

- $G_{7}$ corresponding to the static spacetimes Einstein and the anti Einstein universe, and one non-static spacetime.

- $G_{6}$ corresponding to the static spacetimes Bertotti-Robinson and two other metrics of Petrov type $\mathrm{D}$, and six non-static spacetimes.

- $G_{4}$ is a class of metrics involving one or two arbitrary functions of one variable.

Azad et al. [4] applied Lie group analysis to study the wave equation on the classes of static spherically symmetric spacetimes admitting the isometry groups $G_{10}$ or $G_{7}$ or $G_{6}$. The Iwasawa decomposition for the symmetry algebras was obtained to partially classify non-conjugate solvable algebras. The optimal system of subalgebras was not given in this previous study.

The $G_{7}$ spacetimes admit either $s o(4) \oplus \mathbb{R}$ or $s o(1,3) \oplus \mathbb{R}$ as isometry algebras as shown in [3]. In this paper, we continue the investigation started in [4] by finding the optimal system of subalgebras of dimension of at most three and the corresponding invariant solutions for spacetimes admitting $G_{7}$ as isometry algebras. We expect these solutions to be of interest to mathematical physicists.

As regards optimal systems, we can always construct a family of group invariant solutions obtained by using a subgroup of a symmetry group admitted by a given differential equation, as explained in [5]. Since there are infinitely many subgroups of a symmetry group admitted by a given differential equation, listing of all the group invariant solutions is impossible. However, obtaining 
optimal systems-meaning conjugacy classes- of s-dimensional subgroups of the symmetry group and applying the optimal systems leads to an effective and systematic mechanism of classifying the group invariant solutions. This leads to non-similar invariant solutions under symmetry transformations.

Classifying the group invariant solutions by utilizing optimal systems is a significant application of Lie group and Lie symmetry methods to differential equations. The method was first introduced by Ovsiannikov [6]. He applied this method in classifying the invariant solutions of the one-dimensional gasdynamic equation [7]. Ibragimov extended this work to the two-dimensional adiabatic gas motions in his master thesis [8] by applying the expansion method for solvable Lie algebra. The main idea behind the method is discussed in detail in Ibragimov [5,9], Olver [10] and Hydon [11].

The symmetry Lie algebra of the equations under study is non-solvable, but finding the optimal systems for non-solvable Lie algebras is more challenging. In this paper, improved algorithms are introduced and applied to construct the optimal systems of dimension of at most three of Lie algebras. The reason is that a PDE with four independent variables can be reduced to an ordinary differential equation (ODE) using three-dimensional subalgebras satisfying the transversality condition with rank three [10]. This provides the non-trivial invariant solutions under a maximum number of symmetries.

The paper is organized as follows: in Section 2, algorithms to construct the optimal systems of dimension of at most three of Lie algebras are introduced. In Section 3, Lie point symmetry transformations of the wave equation on the metrics considered in this paper are found. In Section 4, the algorithms are applied to determine the Lie algebra structure and optimal systems of the symmetries. In Section 5, joint invariants and invariant solutions corresponding to three-dimensional optimal systems are determined.

\section{Algorithms to Construct the Optimal Systems of Dimension of at Most Three of Non-Solvable Lie Algebras}

In this paper, we are interested only in finding an optimal system of subalgebras of dimension of at most three as explained in the introduction. This is achieved by using the algorithms explained below. These algorithms are based on a combination of the expansion method and algorithms for determining maximal solvable subalgebras of semi-simple Lie algebras.

If $X$ is a solvable, then either $X$ is abelian or it can be obtained from its commutator $X^{\prime}$ by a sequence of one-dimensional ideals . Thus, in any case, by using normalizers or centralizers, one can reach $X$ from lower dimensional subalgebras. In more detail, the expansion method is revised and improved to a systematic method by using the normalizers and their associated quotient algebras as follows:

Let $\Theta_{r}$ be the optimal systems of $r$-dimensional solvable subalgebras of the solvable algebra $\mathcal{L}$. For every $X \in \Theta_{t-1}$, find the normalizer $\mathcal{N}(X)$. In case the quotient algebra $\mathcal{N}(X) / X$ is non-zero, we find a one-dimensional optimal system in $\mathcal{N}(X) / X$ for every $X \in \Theta_{t-1}$ by considering the invariants of the adjoint representation of $\mathcal{N}(X) / X$.

Among the constructed optimal systems of $\mathcal{N}(X) / X$ for every $X \in \Theta_{t-1}$, we may still have repetitions in their preimages in $\mathcal{L}$. Removing the repetitions provides an optimal system $\Theta_{t}$. Enumeration of all non-conjugate solvable subalgebras of $\mathcal{L}$ can finally be done through consecutive choice of the values of $t$ from 1 till $\operatorname{dim}(\mathcal{L})$.

The Expansion method can be used to find optimal systems of solvable subalgebras in solvable or non-solvable Lie algebras. However, dealing with the general adjoint action of the group once the Lie algebra is non-solvable is very difficult. Therefore, in order to find the optimal systems of solvable subalgebras in a non-solvable Lie algebra, we proceed as follows:

For a general Lie algebra with Levi decomposition $\mathcal{L}=\mathcal{S} \oplus_{S} \mathscr{R}(\mathcal{L})$, where $\mathcal{S}$ is a semisimple subalgebra of $\mathcal{L}$ and $\mathscr{R}(\mathcal{L})$ is the radical of $\mathcal{L}$, every maximal solvable subalgebra is of the form $\mathcal{M} \oplus_{\mathcal{S}} \mathscr{R}(\mathcal{L})$, where $\mathcal{M}$ is maximal solvable in $\mathcal{S}$. The maximal solvable subalgebras can be determined using the algorithms given in [12] or more efficiently using the method detailed in Section 2.1. For a semisimple algebra $\mathcal{S}$, there is a subalgebra $\mathcal{N}$ in which all elements are ad-nilpotent and which 
contains-up to conjugacy-all the commutators of solvable subalgebras of $\mathcal{S}$. All the maximal solvable subalgebras that are not compact tori can be constructed from the normalizers of conjugacy classes in $\mathcal{N}$-as detailed in Section 2.1. Then, the task of finding the optimal systems of solvable subalgebras of the Lie algebra $\mathcal{L}$ is reduced to finding the optimal systems of solvable subalgebras in each of these maximal solvable subalgebras using the expansion method. Finally, the repetitions in the obtained rough classification of subalgebras are removed using the adjoint representation of $\mathcal{L}$.

As a special case, if the radical is the center, then the calculations are greatly simplified. The reason is that it is enough to find the optimal systems of solvable subalgebras in each conjugacy class of maximal solvable subalgebras in the semisimple part of $\mathcal{L}$. Then, the repetitions in the obtained rough classification of subalgebras are removed using the adjoint representation of the semisimple part of $\mathcal{L}$. Finally, adjoining the subalgebras of the radical gives the optimal systems of solvable subalgebras in $\mathcal{L}$.

In order to find the general adjoint action of the semisimple part of $\mathcal{L}$, we need to make a suitable change of basis depending on the root space decomposition or the Iwasawa decomposition of the semisimple part $\mathscr{L}$ based on the signature of the Killing form.

\subsection{Algorithm for Finding the Conjugacy Classes of Maximal Solvable Subalgebras}

For the convenience of the reader who is not a specialist in Lie theory, we first recall how to construct Cartan algebras and roots algorithmically from a knowledge of the commutator table of a given Lie algebra.

The structure of a semisimple Lie algebra is determined by its roots. For more details, the reader is referred to [13]; see also [14-16].

Definition 1. A Lie subalgebra $H$ of a Lie algebra $\mathcal{L}$ is said to be a Cartan subalgebra if $H$ is abelian and every element $h \in H$ is semisimple: by a semisimple element, we mean an element that is diagonalizable in the adjoint representation. Moreover, $H$ is maximal with these properties.

Definition 2. Let $C$ be a Cartan subalgebra of a semisimple Lie algebra $\mathcal{L}$. A non-zero vector $v \in \mathcal{L}^{\mathbb{C}}:=\mathcal{L}+i \mathcal{L}$ such that $[h, v]=\lambda(h) v$ for all $h \in C$ is called a root vector and the corresponding linear function $\lambda$ is called a root of the Cartan algebra $C$.

In general, the roots will be complex-valued. In the following argument, we will use the notion of positive roots, so one needs to define what it means for a complex valued root to be positive.

Definition 3. A complex number $z=a+i b, a, b \in \mathbb{R}$ is positive if either its real part $a$ is positive or $a=0$, but its imaginary part $b$ is positive.

Fix a basis $h_{1}, \ldots, h_{r}$ of a Cartan algebra $C$. A non-zero root $\lambda$ is positive if the first non-zero number $\lambda\left(h_{i}\right)$ is a complex positive number. Otherwise, it is called a negative root. Positive roots which are not a sum of two positive roots are called simple roots.

The well known software Maple is able to find the root space decompositions of Lie algebras of fairly high dimensions by using the command "RootSpaceDecomposition(C)", where $C$ is a list of vectors in a Lie algebra, defining a Cartan subalgebra.

The Cartan algebra is picked up using an algorithm due to de Graaf [15]. However, one gets better coordinates for computation if one chooses a Cartan algebra by enlarging a given diagonalizable subalgebra to a Cartan subalgebra following the algorithms given in [13]. We need in this paper only a special case of these algorithms to compute the Cartan subalgebras. We first compute the Killing form of the Lie algebra. If it is negative definite, pick any non-zero element $X$ and compute its centralizer. By a negative definite matrix, we mean a matrix which is equal to its conjugate transpose and its eigenvalues are strictly negative. If the centralizer of $X, \mathcal{C}(X)$, is self centralizing, i.e., $\mathcal{C}(\mathcal{C}(X))=\mathcal{C}(X)$, then $\mathcal{C}(X)$ is the Cartan subalgebra. Otherwise, we can find a linearly independent element $Y$ in the centralizer of $X$. Continue this procedure with the abelian algebra $\langle X, Y\rangle$ until a self centralizing 
subalgebra is reached. The obtained algebra is the Cartan algebra because it is abelian and every element is diagonalizable.

On the other hand, if the Killing form is not negative definite and a maximal compact subalgebra is known, say $K$, then computing a Cartan subalgebra $C$ of $K$ using the procedure explained in the previous paragraph for compact algebras and the centralizer of $C$ in the full Lie algebra gives us the required Cartan algebra.

The main use of Cartan algebras is to find all the maximal solvable subalgebras [17]. In case the Lie algebra $\mathcal{L}$ is compact, a Cartan algebra is, up to conjugacy, the only maximal solvable subalgebra. This follows from Lie's theorem on solvable algebras [14].

There is a solvable subalgebra $B$ with real eigenvalues in the adjoint representation of $\mathcal{L}$ with the property that any other solvable algebra with real eignvalues in the adjoint representation is conjugate to a subalgebra of $B$.

In [12], it is found that the algebra $B$ can be constructed algorithmically by using positive roots of a given maximally real Cartan subalgebra; by maximally real Cartan subalgebra, we mean a Cartan algebra whose real part has maximal possible dimension. In case the Killing form is not negative definite, any Cartan algebra is a sum of two subalgebras such that one of them has all real eigenvalues in the adjoint representation in $\mathcal{L}$ and the other has all purely imaginary eigenvalues in the adjoint representation in $\mathcal{L}$. We call the first subalgebra the real part of the Cartan subalgebra and the second subalgebra the compact part of the Cartan subalgebra. Let $N$ be the algebra consisting of the real and imaginary parts of the positive root vectors for the given maximally real Cartan subalgebra. Then, the algebra $B=A+N$ where $A$ is the real part of the maximally real Cartan subalgebra has the property that every solvable algebra with real eigenvalues in the adjoint representation is conjugate to subalgebra of $B$. Moreover, all maximal solvable algebras which are non-abelian can be obtained by computing normalizers of subalgebras of $N$. In more detail, we consider conjugacy classes of subalgebras of $N$. If $X$ is a representative of such a class, we compute the normalizer of $X$ and its Levi decomposition. We keep only those $X$ in which the normalizer of $X$ has Levi decomposition $\mathcal{N}(X)=S+\mathcal{R}(\mathcal{N}(X)), \mathcal{R}(\mathcal{N}(X)) / X$ a torus and where the semisimple part has a compact Cartan subalgebra. If $T$ is this compact Cartan subalgebra, then $T+\mathcal{R}(\mathcal{N}(X))$ is a maximal solvable subalgebra and all such, apart from compact maximal tori-if any- are obtained in this way.

\subsection{Algorithm for Finding Three-Dimensional Optimal System of Non-Solvable Subalgebras of a Lie Algebra}

- It is a classical fact that any non-solvable three-dimensional subalgebra is isomorphic to either $s l(2, \mathbb{R})$ or $s o(3)$ copies in $\mathscr{L}$ up to conjugacy where $\mathscr{L}$ is a semisimple subalgebra of the given Lie algebra $\mathcal{L}$. Therefore, one can construct the three-dimensional optimal system of non-solvable subalgebras by finding copies of $s o(3)$ and $\operatorname{sl}(2, \mathbb{R})$ in $\mathscr{L}$.

- In order to find such copies in the semisimple Lie algebra $S$, we have developed the following algorithms which are based on the canonical relations for so(3):

$$
[X, A]=Y, \quad[A, Y]=X, \quad[Y, X]=A,
$$

and $\operatorname{sl}(2, \mathbb{R})$

$$
[A, B]=2 B, \quad[A, Y]=-2 Y, \quad[B, Y]=A .
$$

To find the non-conjugate copies of so(3):

- We start with an element $A$ of the one-dimensional optimal system of $S$ whose non-zero eigenvalues in the adjoint representation are purely imaginary.

- By scaling, we may assume that this eigenvalue is $i$. Let $X+i Y$ be the eigenvector of $A$ corresponding to the eigenvalue $i$. If $[X, Y]=\lambda A$ for some negative constant $\lambda$, then the algebra $\langle A, X, Y\rangle$ forms a copy of $s o(3)$. 
- Applying this algorithm for all elements in the one-dimensional optimal system gives us the copies of so(3).

- Removing the repetitions using invariant tools gives the non-conjugate copies of $s o(3)$.

To find the copies of $\operatorname{sl}(2, \mathbb{R})$ :

- We start with an element of the two-dimensional optimal system of non-abelian subalgebras.

- If $\langle A, B\rangle$ is such algebra with $[A, B]=c B$ for some non-zero constant $c$, find the eigenvectors of $\operatorname{ad} A$, if any corresponding to the eigenvalue $-c$. We reject $\langle A, B\rangle$ if there is no such eigenvalue. Otherwise, let $Y$ be an eigenvector of $\operatorname{ad}(A)$ with eigenvalue $-c$. If the commutator $[B, Y]$ is a nonzero multiple of $A$, then $\langle A, B, Y\rangle$ is a copy of $\operatorname{sl}(2, \mathbb{R})$.

- Removing the repetitions using invariant tools gives the non-conjugate copies of $\operatorname{sl}(2, \mathbb{R})$.

\section{Lie Point Symmetry Transformations of the Wave Equation}

The wave equation on a spacetime is given by $\square_{g} u=0$, where $\square_{g}=\frac{\partial}{\partial x_{i}}\left(\sqrt{|g|} g^{i k} \frac{\partial}{\partial x_{k}}\right)$ is called the Laplace-Beltrami operator for the metric given by

$$
d s^{2}=e^{v(r, t)} d t^{2}-e^{\lambda(r, t)} d r^{2}-e^{\mu(r, t)} d \theta^{2}-e^{\mu(r, t)} \sin ^{2} \theta d \varphi^{2} .
$$

Hence, the wave equation $\square_{g} u=0$ on the metric (3) can be written as

$$
\frac{\partial}{\partial t}\left(e^{\left(\mu-\frac{v}{2}+\frac{\lambda}{2}\right)} \sin \theta \frac{\partial u}{\partial t}\right)-\frac{\partial}{\partial r}\left(e^{\left(\mu+\frac{v}{2}-\frac{\lambda}{2}\right)} \sin \theta \frac{\partial u}{\partial r}\right)-\frac{\partial}{\partial \theta}\left(e^{\left(\frac{v}{2}+\frac{\lambda}{2}\right)} \sin \theta \frac{\partial u}{\partial \theta}\right)-\frac{\partial}{\partial \varphi}\left(\frac{e^{\left(\frac{v}{2}+\frac{\lambda}{2}\right)}}{\sin \theta} \frac{\partial u}{\partial \varphi}\right)=0 .
$$

The approach to find the symmetries of the wave equation using the conformal Killing vector field of the underlying spacetimes metric is due to Yuri Bozhkov and Igor Leite Freire [18].

Theorem 1 ([18]). Let $M^{n}$ be a Lorentzian manifold of dimension $n \geq 3$ with the metric $g$ given in local coordinates $\left\{x_{1}, x_{2}, \ldots, x_{n}\right\}$. The Lie symmetries of wave equation $\square_{g} u=0$ on $M^{n}$ have the form

$$
X=\xi^{i}(x) \frac{\partial}{\partial x_{i}}+\left(\left(\frac{2-n}{4} \mu(x)+c\right) u+b(x)\right) \frac{\partial}{\partial u^{\prime}}
$$

where $c$ is an arbitrary constant,

$$
\square_{g} b(x)=0, \quad \square_{g} \mu(x)=0,
$$

$Y=\xi^{i}(x) \frac{\partial}{\partial x_{i}}$ is a conformal Killing vector field of the metric $g$ such that

$$
\left(£_{Y} g\right)_{a b}=\xi^{c} \partial_{c} g_{a b}+g_{c b} \partial_{a} \xi^{c}+g_{c a} \partial_{b} \xi^{c}=\mu(x) g_{a b}
$$

and $E_{Y}$ denotes the Lie derivative with respect to vector field $Y$, where $b(x)$ and $\mu(x)$ satisfy (6).

\subsection{Lie Point Symmetry Transformations of the Wave Equation on Einstein Spacetime}

The wave equation $\square_{g} u=0$ on the spherically symmetric space admitting $s o(4) \oplus \mathbb{R}$ as isometry algebra can be obtained from Equation (4) by substituting $v=0, \lambda=-\ln \left(\alpha r^{2}+1\right)$ and $\mu=\ln r^{2}$, $\alpha=-c^{2}<0$ as shown in [3].

From now on, we will work with Cartesian coordinates as their introduction simplifies many comutations. The wave equation under study can be written in Cartesian coordinates $x=r \cos \varphi \sin \theta, y=r \sin \varphi \sin \theta, z=r \cos \theta$ as:

$$
\begin{aligned}
& u_{t t}+\left(c^{2} x^{2}-1\right) u_{x x}+\left(c^{2} y^{2}-1\right) u_{y y}+\left(c^{2} z^{2}-1\right) u_{z z}+3 c^{2} z u_{z}+2 c^{2} x z u_{z x} \\
& +3 c^{2} x u_{x}+2 c^{2} x y u_{x y}+2 c^{2} y z u_{y z}+3 c^{2} y u_{y}=0 .
\end{aligned}
$$


By using Theorem 1 and the isometries of the metric given in [3], the Lie symmetry algebra of the wave Equation (8) consists of the eight-dimensional subalgebra spanned by

$$
\begin{array}{llll}
X_{1}=B \frac{\partial}{\partial y}, & X_{2}=B \frac{\partial}{\partial x}, & X_{3}=B \frac{\partial}{\partial z}, & X_{4}=x \frac{\partial}{\partial z}-z \frac{\partial}{\partial x}, \\
X_{5}=z \frac{\partial}{\partial y}-y \frac{\partial}{\partial z}, & X_{6}=x \frac{\partial}{\partial y}-y \frac{\partial}{\partial x}, & X_{7}=\frac{\partial}{\partial t}, & X_{8}=u \frac{\partial}{\partial u},
\end{array}
$$

and the infinite-dimensional ideal consisting of the operators

$$
X_{\tau}=\tau(t, x, y, z) \frac{\partial}{\partial u},
$$

where $\tau(t, x, y, z)$ is an arbitrary solution of the wave Equation (8) and $B=\sqrt{1-c^{2}\left(x^{2}+y^{2}+z^{2}\right)}$.

Moreover, the one-parameter groups $G_{i}(\varepsilon)=\left\{e^{\varepsilon X_{i}}, \varepsilon \in \mathbb{R}\right\}$ generated by (9) are given as follows:

$$
\begin{aligned}
& G_{1}\left(\varepsilon_{1}\right):(t, x, y, z, u) \mapsto\left(t, x, \frac{1}{c} \sqrt{1-c^{2}\left(x^{2}+z^{2}\right)} \sin \left(\arctan \left(\frac{c y}{B}\right)+c \varepsilon_{1}\right), z, u\right), \\
& G_{2}\left(\varepsilon_{2}\right):(t, x, y, z, u) \mapsto\left(t, \frac{1}{c} \sqrt{1-c^{2}\left(y^{2}+z^{2}\right)} \sin \left(\arctan \left(\frac{c x}{B}\right)+c \varepsilon_{2}\right), y, z, u\right), \\
& G_{3}\left(\varepsilon_{3}\right):(t, x, y, z, u) \mapsto\left(t, x, y, \frac{1}{c} \sqrt{1-c^{2}\left(x^{2}+y^{2}\right)} \sin \left(\arctan \left(\frac{c z}{B}\right)+c \varepsilon_{3}\right), u\right), \\
& G_{4}\left(\varepsilon_{4}\right):(t, x, y, z, u) \mapsto\left(t, x \sin \varepsilon_{4}-z \cos \varepsilon_{4}, y, x \sin \varepsilon_{4}+z \cos \varepsilon_{4}, u\right), \\
& G_{5}\left(\varepsilon_{5}\right):(t, x, y, z, u) \mapsto\left(t, x,-z \sin \varepsilon_{5}-y \cos \varepsilon_{5}, y \sin \varepsilon_{5}-z \cos \varepsilon_{5}, u\right), \\
& G_{6}\left(\varepsilon_{6}\right):(t, x, y, z, u) \mapsto\left(t,-y \sin \varepsilon_{6}+x \cos \varepsilon_{5}, x \sin \varepsilon_{6}+y \cos \varepsilon_{6}, z, u\right), \\
& G_{7}\left(\varepsilon_{7}\right):(t, x, y, z, u) \mapsto\left(t+\varepsilon_{7}, y, z, u\right), \\
& G_{8}\left(\varepsilon_{8}\right):(t, x, y, z, u) \mapsto\left(t, x, y, z, u+\varepsilon_{8}\right) .
\end{aligned}
$$

\subsection{Lie Point Symmetry Transformations of the Wave Equation on Anti-Einstein Spacetime}

The wave Equation $\square_{g} u=0$ on the spherically symmetric space admitting $s o(1,3) \oplus \mathbb{R}$ as isometry algebra can be obtained from Equation (4) by substituting $v=0, \lambda=-\ln \left(\alpha r^{2}+1\right)$ and $\mu=\ln r^{2}, \alpha=c^{2}>0$ as shown in [3].

As before, we will work with Cartesian coordinates as their introduction simplifies many comutations. The wave equation under study can be written in Cartesian coordinates $x=r \cos \varphi \sin \theta$, $y=r \sin \varphi \sin \theta, z=r \cos \theta$ as:

$$
\begin{aligned}
& \left(c^{2} x^{2}+1\right) u_{x x}+\left(c^{2} y^{2}+1\right) u_{y y}+\left(c^{2} z^{2}+1\right) u_{z z}+2 c^{2} y z u_{y z} \\
& +3 x c^{2} u_{x}+2 c^{2} x y u_{x y}+2 c^{2} x z u_{x z}+3 c^{2} z u_{z}+3 c^{2} y u_{y}-u_{t t}=0 .
\end{aligned}
$$

By using Theorem 1 and the isometries of the metric given in [3], the Lie symmetry algebra of the wave Equation (12) consists of the eight-dimensional subalgebra spanned by

$$
\begin{array}{llll}
X_{1}=B \frac{\partial}{\partial y}, & X_{2}=B \frac{\partial}{\partial x}, & X_{3}=B \frac{\partial}{\partial z}, & X_{4}=x \frac{\partial}{\partial z}-z \frac{\partial}{\partial x}, \\
X_{5}=z \frac{\partial}{\partial y}-y \frac{\partial}{\partial z}, & X_{6}=x \frac{\partial}{\partial y}-y \frac{\partial}{\partial x}, & X_{7}=\frac{\partial}{\partial t}, & X_{8}=u \frac{\partial}{\partial u},
\end{array}
$$

and the infinite-dimensional ideal consisting of the operators

$$
X_{\tau}=\tau(t, x, y, z) \frac{\partial}{\partial u},
$$

where $\tau(t, x, y, z)$ is an arbitrary solution of the wave Equation (12) and $B=\sqrt{1+c^{2}\left(x^{2}+y^{2}+z^{2}\right)}$. 
Moreover, the one-parameter groups $G_{i}(\varepsilon)=\left\{e^{\varepsilon X_{i}}, \varepsilon \in \mathbb{R}\right\}$ generated by (13) are given as follows:

$$
\begin{aligned}
& G_{1}\left(\varepsilon_{1}\right):(t, x, y, z, u) \mapsto\left(t, x, \frac{e^{-c \varepsilon_{1}}\left(2 c B e^{2 c \varepsilon_{1}} y+\left(B^{2}+c^{2} y^{2}\right) e^{2 c \varepsilon_{1}}-1-c^{2}\left(x^{2}+z^{2}\right)\right)}{2 c^{2} y+2 c B}, z, u\right), \\
& G_{2}\left(\varepsilon_{2}\right):(t, x, y, z, u) \mapsto\left(t, \frac{\left(2 \mathrm{e}^{2 c \varepsilon_{2}}\left(B^{2}-1\right)+2 c B \mathrm{e}^{2 c \varepsilon_{2}} x-c^{2}\left(y^{2}+z^{2}\right)+\mathrm{e}^{2 c \varepsilon_{2}}-1\right) \mathrm{e}^{-c \varepsilon_{2}}}{2 c^{2} x+2 c B}, y, z, u\right), \\
& G_{3}\left(\varepsilon_{3}\right):(t, x, y, z, u) \mapsto\left(t, x, y, \frac{\mathrm{e}^{-c \varepsilon_{3}}\left(2 c B \mathrm{e}^{2 c \varepsilon_{3}} z+B^{2} \mathrm{e}^{2 c c_{3}}-1-c^{2}\left(x^{2}+y^{2}\right)\right)}{2 c^{2} z+2 c B}, u\right), \\
& G_{4}\left(\varepsilon_{4}\right):(t, x, y, z, u) \mapsto\left(t, x \sin \varepsilon_{4}-z \cos \varepsilon_{4}, y, x \sin \varepsilon_{4}+z \cos \varepsilon_{4}, u\right), \\
& G_{5}\left(\varepsilon_{5}\right):(t, x, y, z, u) \mapsto\left(t, x,-z \sin \varepsilon_{5}-y \cos \varepsilon_{5}, y \sin \varepsilon_{5}-z \cos \varepsilon_{5}, u\right), \\
& G_{6}\left(\varepsilon_{6}\right):(t, x, y, z, u) \mapsto\left(t,-y \sin \varepsilon_{6}+x \cos \varepsilon_{5}, x \sin \varepsilon_{6}+y \cos \varepsilon_{6}, z, u\right), \\
& G_{7}\left(\varepsilon_{7}\right):(t, x, y, z, u) \mapsto\left(t+\varepsilon_{7}, x, y, z, u\right), \\
& G_{8}\left(\varepsilon_{8}\right):(t, x, y, z, u) \mapsto\left(t, x, y, z, u+\varepsilon_{8}\right) .
\end{aligned}
$$

\section{Lie Algebra Structure and Optimal Systems}

\subsection{Lie Point Symmetry Algebra of the Wave Equation on Einstein Spacetime}

The non-zero Lie brackets of (9) are:

$$
\begin{array}{llll}
{\left[X_{1}, X_{2}\right]=c^{2} X_{6},} & {\left[X_{1}, X_{3}\right]=c^{2} X_{5},} & {\left[X_{1}, X_{5}\right]=-X_{3},} & {\left[X_{1}, X_{6}\right]=-X_{2},} \\
{\left[X_{2}, X_{3}\right]=-c^{2} X_{4},} & {\left[X_{2}, X_{4}\right]=X_{3},} & {\left[X_{2}, X_{6}\right]=X_{1},} & {\left[X_{3}, X_{4}\right]=-X_{2},} \\
{\left[X_{3}, X_{5}\right]=X_{1},} & {\left[X_{4}, X_{5}\right]=X_{6},} & {\left[X_{4}, X_{6}\right]=-X_{5},} & {\left[X_{5}, X_{6}\right]=X_{4} .}
\end{array}
$$

The Levi-Decomposition of this algebra is $\mathcal{L}=\left\{X_{1}, X_{2}, X_{3}, X_{4}, X_{5}, X_{6}\right\} \oplus\left\{X_{7}, X_{8}\right\}$. Let $S$ be the semisimple part. To identify the semisimple part, we need to find a Cartan algebra and the corresponding root space decomposition.

First of all, after computing the Killing form, we see that it is negative definite. Thus, to determine a Cartan algebra, choose any non-zero element in the semisimple part $S$. We choose, for example, the element $X_{3}$ and compute its centralizer. The centralizer turns out to be $\left\{X_{3}, X_{6}\right\}$ and the subalgebra $\left\{X_{3}, X_{6}\right\}$ is self centralizing. Thus, $C=\left\{X_{3}, X_{6}\right\}$ is a Cartan subalgebra which is itself the only maximal solvable subalgebra up to the conjugacy as mentioned in Section 2.1. The roots for this Cartan subalgebra are $\{(c i, i),(-c i, i),(-c i,-i),(c i,-i)\}, i=\sqrt{-1}$. Therefore, the positive roots are $\{(c i, i),(c i,-i)\}$. The root vectors for the positive roots are $\left\{X_{1}+c X_{4}+i\left(X_{2}+c X_{5}\right), X_{1}-c X_{4}+i\left(X_{2}-\right.\right.$ $\left.\left.c X_{5}\right)\right\}$. Since the negative roots are conjugates of the positive roots, the real and the imaginary parts of the positive root vectors must generate, as a Lie algebra, the full Lie algebra.

This gives us the change of basis which gives the general adjoint action of the group of symmetry transformations:

$$
\begin{aligned}
& V_{1}=X_{1}+c X_{4}, \quad V_{2}=X_{2}+c X_{5}, \quad V_{3}=X_{3}-c X_{6}, \quad V_{4}=X_{1}-c X_{4}, \\
& V_{5}=X_{2}-c X_{5}, \quad V_{6}=X_{3}+c X_{6}, \quad V_{7}=X_{7}, \quad V_{8}=X_{8} .
\end{aligned}
$$

The corresponding non-zero Lie brackets of this subalgebra are:

$$
\begin{array}{cll}
{\left[V_{1}, V_{2}\right]=-2 c V_{3}} & {\left[V_{1}, V_{3}\right]=2 c V_{2},} & {\left[V_{2}, V_{3}\right]=-2 c V_{1}, \quad\left[V_{4}, V_{5}\right]=2 c V_{6},} \\
& {\left[V_{4}, V_{6}\right]=-2 c V_{5},} & {\left[V_{5}, V_{6}\right]=2 c V_{4} .}
\end{array}
$$

It is obvious from (18) that the subalgebra $\left\langle V_{1}, V_{4}\right\rangle$ is Cartan since it is abelian and it is self centralizing. Since our Lie algebra is compact, therefore, $\left\langle V_{1}, V_{4}\right\rangle$ is the only maximal solvable algebra up to the conjugacy. The subalgebra $\left\langle V_{1}, V_{4}\right\rangle$ is conjugate to $\left\langle V_{3}, V_{6}\right\rangle$.

As we will see later, $\left\langle V_{1}, V_{2}, V_{3}\right\rangle$ and $\left\langle V_{4}, V_{5}, V_{6}\right\rangle$ form two copies of so(3) which commute with each other. Since so(4), which is the set of all skew symmetric $4 \times 4$ matrices, is also isomorphic to $s o(3) \oplus s o(3)$ [19], we see that the semisimple part is isomorphic to $s o(4)$. This decomposition can be 
obtained by working with a Cartan subalgebra of so(4) and determining its root space decomposition as was done above.

\subsubsection{Optimal Systems of Solvable Subalgebras of so(4)}

To find the optimal systems of $s o(4)$, we first find the one-dimensional optimal system and a rough classification of higher order subalgebras inside the maximal solvable subalgebra $\left\langle V_{1}, V_{4}\right\rangle$ which is abelian. Secondly, we obtain the higher-dimensional optimal system by removing the repetitions from the obtained rough classification of subalgebras using the adjoint representation of so(4).

Theorem 2. The optimal systems $\Theta_{i}$ up to order three of solvable subalgebras of so(4) with the non-zero Lie brackets (18) are the following:

- The one-dimensional optimal system $\Theta_{1}$ is $\left\{\left\langle V_{1}+\alpha V_{4}\right\rangle,\left\langle V_{1}\right\rangle,\left\langle V_{4}\right\rangle, \alpha \neq 0\right\}$,

- The two-dimensional optimal system $\Theta_{2}$ is $\left\{\left\langle V_{1}, V_{4}\right\rangle\right\}$.

There is no three-dimensional optimal system.

Proof. Clearly, it is enough to deal with the one-dimensional optimal system only. The one-dimensional optimal system of the maximal solvable subalgebra of so(4) is itself the one-dimensional optimal system of so(4). This is because the representative elements are non-conjugate under the adjoint representation of $s o(4)$ given by

$$
A\left(\varepsilon_{1}, \ldots, \varepsilon_{6}\right)=e^{\varepsilon_{1} C(1)} \ldots e^{\varepsilon_{6} C(6)},
$$

where $C(j)$ is the matrix whose $(i, k)^{t h}$ entries are given as $c_{i j}^{k}$ : here, the constants $c_{i j}^{k}$ are the structure constants relative to the basis $V_{1}, \ldots, V_{6}$.

4.1.2. Optimal Systems of Solvable Subalgebras of $\mathcal{L}=s o(4) \oplus \mathbb{R}^{2}$

First, note the general fact that if $\mathcal{L}=S \oplus R$ where $S$ is the semisimple part and the radical $R$ is the center, then the conjugacy classes of $S$ can be joined with elements of the center to obtain conjugacy classes of $\mathcal{L}$, as follows:

Let $\pi: S \oplus R \rightarrow S$ be the projection defined by $\pi(x, y)=x$. This is a homomorphism because $R$ is an ideal. Therefore, it will map conjugate classes to conjugate classes.

Every $k$-dimensional subalgebra of $\mathcal{L}$ is of the form $\left\langle x_{1}+y_{1}, x_{2}+y_{2}, \ldots, x_{k}+y_{k}\right\rangle$, where $x_{i} \in S$, $y_{i} \in R$. Its projection is $\left\langle x_{1}, \ldots, x_{k}\right\rangle$ of dimension less than or equal to $k$. Moreover, if $\left\langle x_{1}+y_{1}, x_{2}+\right.$ $\left.y_{2}, \ldots, x_{k}+y_{k}\right\rangle$ is conjugate to $\left\langle\tilde{x_{1}}+\tilde{y_{1}}, \tilde{x_{2}}+\tilde{y_{2}}, \ldots, \tilde{x_{k}}+\tilde{y_{k}}\right\rangle$, then as the radical $R$ is the center, $y_{i}=\tilde{y_{i}}$ and $\left\langle x_{1}, x_{2}, \ldots, x_{k}\right\rangle$ is conjugate to $\left\langle\tilde{x_{1}}, \tilde{x_{2}}, \ldots, \tilde{x}_{k}\right\rangle$. However, the dimension of the image algebra of $\left\langle\tilde{x_{1}}+\tilde{y_{1}}, \tilde{x_{2}}+\tilde{y_{2}}, \ldots, \tilde{x_{k}}+\tilde{y_{k}}\right\rangle$ can go down. Thus, to get all conjugacy classes of the full algebra, we start with the elements of the optimal systems of $S$ and add to each one of them arbitrary elements of the center and keep those that form a subalgebra. The classes of the center correspond to the zero subspace of $S$. This will give all the conjugacy classes of the full algebra. Applying this to $\mathcal{L}=s o(4) \oplus \mathbb{R}^{2}$, we obtain the following classes.

Clearly, the one-dimensional optimal system $\tilde{\Theta}_{1}$ of $\mathbb{R}^{2}$ is $\left\{\left\langle V_{7}\right\rangle,\left\langle V_{8}\right\rangle,\left\langle V_{7}+\alpha V_{8}\right\rangle, \alpha \neq 0\right\}$ and the only two-dimensional optimal system $\tilde{\Theta}_{2}$ of $\mathbb{R}^{2}$ is $\left\{\left\langle V_{7}, V_{8}\right\rangle\right\}$.

In order to get the optimal systems of the full Lie algebra up to order three, we use the optimal systems of so(4) constructed in Theorem 2 . We join it with the optimal system of the abelian algebra $\mathbb{R}^{2}$ as explained above.

- To get the one-dimensional optimal system of $\mathcal{L}$, we have the cases:

1. We add an arbitrary element from $\mathbb{R}^{2}$ to every element in $\Theta_{1}$; in this case, we get $\left\{\left\langle V_{1}+\alpha V_{4}+Z_{1}\right\rangle,\left\langle V_{1}+Z_{1}\right\rangle,\left\langle V_{4}+Z_{1}\right\rangle, \alpha \neq 0\right\}$. 
2. We take $\tilde{\Theta}_{1}$ itself; in this case, we get $\left\{\left\langle V_{7}\right\rangle,\left\langle V_{8}\right\rangle,\left\langle V_{7}+\beta V_{8}\right\rangle, \beta \neq 0\right\}$.

- To get the two-dimensional optimal system of $\mathcal{L}$, we have the cases:

1. We add an arbitrary element from $\mathbb{R}^{2}$ to every element in $\Theta_{2}$; in this case, we get $\left\{\left\langle V_{1}+Z_{1}, V_{4}+Z_{2}\right\rangle\right\}$.

2. We add an arbitrary element from $\mathbb{R}^{2}$ to every element in $\Theta_{1}$ and combine the result with an element from $\tilde{\Theta}_{1}$; in this case, we get $\left\{\left\langle V_{1}+\alpha V_{4}+Z_{1}, Z_{3}\right\rangle,\left\langle V_{1}+Z_{1}, Z_{3}\right\rangle,\left\langle V_{4}+Z_{1}, Z_{3}\right\rangle, \alpha \neq 0\right\}$.

3. Take $\tilde{\Theta}_{2}$ itself; in this case, we get $\left\{\left\langle V_{7}, V_{8}\right\rangle\right\}$.

- $\quad$ To get the three-dimensional optimal system of $\mathcal{L}$,

1. Either we add an arbitrary element from $\mathbb{R}^{2}$ to every element in $\Theta_{2}$ and combine the result with an element from $\tilde{\Theta}_{1}$; in this case, we get $\left\{\left\langle V_{1}+Z_{1}, V_{4}+Z_{2}, Z_{3}\right\rangle\right\}$;

2. or we add an arbitrary element from $\mathbb{R}^{2}$ to every element in $\Theta_{1}$ and combine the result with an element from $\tilde{\Theta}_{2}$; in this case, we get $\left\{\left\langle V_{1}+\alpha V_{4}+Z_{1}, V_{7}, V_{8}\right\rangle,\left\langle V_{1}+Z_{1}, V_{7}, V_{8}\right\rangle\right.$, $\left.\left\langle V_{4}+Z_{1}, V_{7}, V_{8}\right\rangle, \alpha \neq 0\right\}$.

Finally, we check that the obtained class is a subalgebra by taking the wedge product of it with its commutator and equate by zero and see if we can kill some constants. This leads to the following theorem:

Theorem 3. The optimal systems of solvable subalgebra of $\mathcal{L}$ with the non-zero Lie brackets (18) are as follows:

- The one-dimensional optimal system is $\left\{\left\langle V_{1}+\alpha V_{4}+Z_{1}\right\rangle,\left\langle V_{1}+Z_{1}\right\rangle,\left\langle V_{4}+Z_{1}\right\rangle,\left\langle V_{7}\right\rangle,\left\langle V_{8}\right\rangle,\left\langle V_{7}+\beta V_{8}\right\rangle\right.$, $\alpha, \beta \neq 0\}$.

- The two-dimensional optimal system is $\left\{\left\langle V_{1}+Z_{1}, V_{4}+Z_{2}\right\rangle,\left\langle V_{1}+\alpha V_{4}+Z_{1}, Z_{3}\right\rangle,\left\langle V_{1}+Z_{1}, Z_{3}\right\rangle,\left\langle V_{4}+Z_{1}\right.\right.$, $\left.\left.Z_{3}\right\rangle,\left\langle V_{7}, V_{8}\right\rangle, \alpha \neq 0\right\}$.

- The three-dimensional optimal system is $\left\{\left\langle V_{1}+Z_{1}, V_{4}+Z_{2}, Z_{3}\right\rangle,\left\langle V_{1}+\alpha V_{4}+Z_{1}, V_{7}, V_{8}\right\rangle,\left\langle V_{1}+Z_{1}\right.\right.$, $\left.\left.V_{7}, V_{8}\right\rangle,\left\langle V_{4}+Z_{1}, V_{7}, V_{8}\right\rangle, \alpha \neq 0\right\}$.

Here, $Z_{1}=\alpha_{1} V_{7}+\beta_{1} V_{8}, Z_{2}=\alpha_{2} V_{7}+\beta_{2} V_{8}$ are arbitrary elements of $\mathbb{R}^{2}$ and $Z_{3}=V_{7}+\alpha_{3} V_{8}$ or $Z_{3}=V_{8}$ represent a one-dimensional optimal system of $\mathbb{R}^{2}$.

4.1.3. Three-dimesional Optimal System of Non-solvable Subalgebras of $\mathcal{L}=s o(4) \oplus \mathbb{R}^{2}$

If $H$ is a three-dimensional non-solvable algebra, then $H$ equals its commutator. As the commutator of $\mathcal{L}$ is so(4), all such subalgebras of $\mathcal{L}$ are subalgebras of so(4). We need to construct the copies of so(3) and $s l(2, \mathbb{R})$, if any, by following the algorithm given in Section 2.2:

- $\quad$ First, construct the copies of $s o(3)$ :

1. The element $V_{1}$ has the eigenvector $V_{2}+i V_{3}$ corresponding to the eigenvalue $2 c i$. Therefore, $\tilde{V}_{1}=\frac{V_{1}}{2 c}$ has the same eigenvector with the eigenvalue $i$. Moreover, $\left[V_{2}, V_{3}\right]=-(2 c)^{2} \tilde{V}_{1}$. Hence, $\left\langle V_{1}, V_{2}, V_{3}\right\rangle$ forms a copy of so(3).

2. The element $V_{4}$ has the eigenvector $V_{5}+i V_{6}$ corresponding to the eigenvalue $-2 c i$. Therefore, $\tilde{V}_{4}=\frac{V_{4}}{-2 c}$ has the same eigenvector with the eigenvalue $i$. Moreover, $\left[V_{5}, V_{6}\right]=-(2 c)^{2} \tilde{V}_{4}$. Hence, $\left\langle V_{4}, V_{5}, V_{6}\right\rangle$ forms a copy of so(3).

3. The element $V_{1}+\alpha V_{4}$ has the eigenvector $V_{2}+V_{5}+i\left(V_{3}-V_{6}\right)$ corresponding to the eigenvalue $2 c i$. Therefore, $\tilde{V}=\frac{V_{1}+\alpha V_{4}}{2 c}$ has the same eigenvector with the eigenvalue $i$. Moreover, and $\left[V_{2}+V_{5}, V_{3}-V_{6}\right]=-2 c \tilde{V}$. Hence, $\left\langle V_{1}+V_{4}, V_{2}+V_{5}, V_{3}-V_{6}\right\rangle$ forms a copy of so(3). Note that here $\alpha$ must be equal to one to ensure that $\left\langle V_{1}+\alpha V_{4}, V_{2}+V_{5}, V_{3}-V_{6}\right\rangle$ is a subalgebra.

- The Lie algebra $s o(4)$ does not contain any copy of $s l(2, \mathbb{R})$, since it does not contain any non-abelian two-dimensional subalgebra. 
This proves the following theorem:

Theorem 4. The three-dimensional optimal system of non-solvable subalgebras of $\mathcal{L}$ is $\left\{\left\langle V_{1}, V_{2}, V_{3}\right\rangle,\left\langle V_{4}, V_{5}, V_{6}\right\rangle,\left\langle V_{1}+V_{4}, V_{2}+V_{5}, V_{3}-V_{6}\right\rangle\right\}$, where the $V_{i}, i=1, \ldots, 6$ form a basis of so(4) given in (17).

\subsection{Lie Point Symmetry Algebra of the Wave Equation on Anti-Einstein Spacetime}

The non-zero Lie brackets of (13) are:

$$
\begin{array}{llll}
{\left[X_{1}, X_{2}\right]=-c^{2} X_{6},} & {\left[X_{1}, X_{3}\right]=-c^{2} X_{5},} & {\left[X_{1}, X_{5}\right]=-X_{3},} & {\left[X_{1}, X_{6}\right]=-X_{2},} \\
{\left[X_{2}, X_{3}\right]=c^{2} X_{4},} & {\left[X_{2}, X_{4}\right]=X_{3},} & {\left[X_{2}, X_{6}\right]=X_{1},} & {\left[X_{3}, X_{4}\right]=-X_{2},} \\
{\left[X_{3}, X_{5}\right]=X_{1},} & {\left[X_{4}, X_{5}\right]=X_{6},} & {\left[X_{4}, X_{6}\right]=-X_{5},} & {\left[X_{5}, X_{6}\right]=X_{4} .}
\end{array}
$$

The Levi-Decomposition of this algebra is $\mathcal{L}=\left\{X_{1}, X_{2}, X_{3}, X_{4}, X_{5}, X_{6}\right\} \oplus\left\{X_{7}, X_{8}\right\}$. Let $S$ be the semisimple part.

To determine the structure of the semisimple part, we need to find a Cartan algebra and the root space decomposition with respect to the Cartan algebra. In this case, the Killing form is not negative definite and it has exactly three negative eigenvalues. This means that the maximal compact algebra is three-dimensional.

The reason is that, if $K$ is a maximal compact subalgebra of the Lie algebra $\mathcal{L}$, then any compact subalgebra of $\mathcal{L}$ is conjugate to a subalgebra of $K$. Moreover, every one-dimensional subalgebra of $K$ is conjugate to a subalgebra of a fixed Cartan subalgebra of $K$ [14-16].

As we will explain later, the subalgebra $\left\langle X_{4}, X_{5}, X_{6}\right\rangle$ is a copy of so(3) in the given Lie algebra. Thus, $K=\left\langle X_{4}, X_{5}, X_{6}\right\rangle$ is a maximal compact subalgebra of the algebra $S$. A Cartan subalgebra of $S$ can be obtained by choosing any element of $K$ and computing its centralizer. We choose, for example, $X_{6}$ as a representative of a Cartan algebra of $K$. Computing the centralizer of $X_{6}$, we find that it is $\left\langle X_{3}, X_{6}\right\rangle$. In addition, as the centralizer of $\left\langle X_{3}, X_{6}\right\rangle$ is itself, $C=\left\langle X_{3}, X_{6}\right\rangle$ is a Cartan subalgebra of $S$. Moreover, computing the eigenvalues of $X_{3}$, we find that all eigenvalues of ad $X_{3}$ are real and $X_{3}$ is diagonalizable. Moreover, the centralizer of $X_{3}$ is $C$ and the centralizer of $X_{6}$ is also $C$; this means that $C$ is the maximally real Cartan subalgebra.

We find roots of $C$ in $S$. The roots are $(c, i),(c,-i),(-c, i),(-c,-i)$, the positive roots are $(c, i),(c,-i)$ and clearly the sum of these positive roots is not a root. The root spaces for the positive roots $(c, i)$ and $(c,-i)$ are $\left\langle X_{1}+c X_{5}+i\left(c X_{4}-X_{2}\right)\right\rangle$. Let $N=\left\langle X_{1}+c X_{5}, X_{2}-c X_{4}\right\rangle$. The algebra $B=A \oplus N$, where $A=\left\langle X_{3}\right\rangle$ is the real part of $C$, has the property that every solvable algebra with real eigenvalues in the adjoint representation is conjugate to a subalgebra of $B$. We compute the normalizers of each conjugacy class of $N$. The normalizer of each representative element of the one-dimensional optimal system of $N$ does not contain a Cartan algebra. Therefore, we keep only $N$ because its normalizer $\mathcal{N}(N)$ is solvable and contains a Cartan algebra. Thus, there is only one maximal solvable subalgebra, namely $\mathcal{N}(N)=\left\langle X_{6}, X_{3}\right\rangle \oplus\left\langle X_{1}+c X_{5}, X_{2}-c X_{4}\right\rangle$. Therfore, the Iwasawa decomposition of $S$ is $K \oplus A \oplus N=\left\langle X_{4}, X_{5}, X_{6}\right\rangle \oplus\left\langle X_{3}\right\rangle \oplus\left\langle X_{1}+c X_{5}, X_{2}-c X_{4}\right\rangle[14,16]$.

This gives us the following change of basis which makes the computations easier:

$$
\begin{aligned}
& V_{1}=X_{4}, \quad V_{2}=X_{5}, \quad V_{3}=X_{6}, \quad V_{4}=X_{3}, \\
& V_{5}=X_{1}+c X_{5}, \quad V_{6}=X_{2}-c X_{4}, \quad V_{7}=X_{7}, \quad V_{8}=X_{8} .
\end{aligned}
$$

The non-zero Lie brackets of (21) are

$$
\begin{array}{llll}
{\left[V_{1}, V_{2}\right]=V_{3},} & {\left[V_{1}, V_{3}\right]=-V_{2},} & {\left[V_{1}, V_{4}\right]=c V_{1}+V_{6},} & {\left[V_{1}, V_{5}\right]=c V_{3},} \\
{\left[V_{1}, V_{6}\right]=-V_{4},} & {\left[V_{2}, V_{3}\right]=V_{1},} & {\left[V_{2}, V_{4}\right]=c V_{2}-V_{5},} & {\left[V_{2}, V_{5}\right]=V_{4},} \\
{\left[V_{2}, V_{6}\right]=c V_{3}} & {\left[V_{3}, V_{5}\right]=V_{6},} & {\left[V_{3}, V_{6}\right]=-V_{5},} & {\left[V_{4}, V_{5}\right]=c V_{5},} \\
{\left[V_{4}, V_{6}\right]=c V_{6} .} & & &
\end{array}
$$


In fact, the semisimple part $S$ is isomorphic to so $(1,3)$ as can be seen by working with its Cartan algebra and the associated root space decompositions. The algebra $\left\langle V_{7}, V_{8}\right\rangle$ is the center of the Lie algebra $\mathcal{L}$.

\subsubsection{Optimal Systems of Solvable Subalgebras of $s o(1,3)$}

To find the optimal system of so(1,3), we first find the one-dimensional optimal system and a rough classification of higher order subalgebras inside the maximal solvable subalgebra spanned by $E_{1}:=V_{3}, E_{2}:=V_{4}, E_{3}:=V_{5}, E_{4}:=V_{6}$. The corresponding non-zero Lie brackets of this subalgebra are:

$$
\left[E_{1}, E_{3}\right]=E_{4}, \quad\left[E_{1}, E_{4}\right]=-E_{3}, \quad\left[E_{2}, E_{3}\right]=c E_{3}, \quad\left[E_{2}, E_{4}\right]=c E_{4} .
$$

Secondly, we obtain the higher-dimensional optimal system by removing the repetitions from the obtained rough classification of subalgebras using the adjoint action of so $(1,3)$.

Theorem 5. The optimal systems $\Theta_{i}$ up to order three of the solvable subalgebras of so $(1,3)$ with the non-zero Lie brackets (22) are the following:

- $\quad$ The one-dimensional solvable optimal system $\Theta_{1}$ is

$\left\{\left\langle V_{4}\right\rangle,\left\langle V_{5}\right\rangle,\left\langle V_{3}+\alpha V_{4}\right\rangle: \alpha \in \mathbb{R}\right\}$.

- The two-dimensional solvable optimal system $\Theta_{2}$ is $\left\{\left\langle V_{3}, V_{4}\right\rangle,\left\langle V_{4}, V_{5}\right\rangle,\left\langle V_{5}, V_{6}\right\rangle\right\}$.

- The three-dimensional solvable optimal system $\Theta_{3}$ is

$\left\{\left\langle V_{4}, V_{5}, V_{6}\right\rangle,\left\langle V_{3}+\alpha V_{4}, V_{5}, V_{6}\right\rangle: \alpha \in \mathbb{R}\right\}$.

Proof. To remove the repetitions in the obtained one-dimensional optimal system and the higher-dimensional rough classification of the maximal solvable subalgebra of $\mathcal{L}$, we use their normalizers in so $(1,3)$ as follows:

- The one-dimensional optimal system of the maximal solvable subalgebra of $s o(1,3)$ is itself the one-dimensional optimal system of $s o(1,3)$. This is because the representative elements are non-conjugate under the adjoint action of $s o(1,3)$, as can be seen using the action of corresponding adjoint group given as in (19).

- The two-dimensional abelian subalgebras are $\left\langle V_{3}, V_{4}\right\rangle,\left\langle V_{5}, V_{6}\right\rangle$. The non-abelian subalgebra $\left\langle V_{4}, V_{5}\right\rangle$ is clearly non-conjugate with both of them. Moreover, since the normalizers of the two-dimensional abelian subalgebras are $\mathcal{N}\left(\left\langle V_{3}, V_{4}\right\rangle\right) /\left\langle V_{3}, V_{4}\right\rangle=0, \mathcal{N}\left(\left\langle V_{5}, V_{6}\right\rangle\right) /\left\langle V_{5}, V_{6}\right\rangle=$ $\left\langle\bar{V}_{3}, \bar{V}_{4}\right\rangle$. As their dimensions are different, they are non-conjugate.

- All the three-dimensional subalgebras given in the rough classification have the same normalizers, centralizers and commutators, namely the abelian subalgebra $\left\langle V_{5}, V_{6}\right\rangle$.

Let $X$ be one of these algebras. We find that the eigenvalues of $X / X^{\prime}$ are repeated real in one case, purely imaginary in one case and complex conjugates but not purely imaginary in the third case. Therefore, they are non-conjugate.

4.2.2. Optimal Systems of Solvable Subalgebras of $\mathcal{L}=s o(1,3) \oplus \mathbb{R}^{2}$

Clearly, the one-dimensional optimal system $\tilde{\Theta}_{1}$ of $\mathbb{R}^{2}$ is $\left\{\left\langle V_{8}\right\rangle,\left\langle V_{7}+\alpha V_{8}\right\rangle: \alpha \in \mathbb{R}\right\}$ and the only two-dimensional optimal system $\tilde{\Theta}_{2}$ of $\mathbb{R}^{2}$ is $\left\{\left\langle V_{7}, V_{8}\right\rangle\right\}$.

In order to get the optimal systems of the full Lie algebra up to order three, we use the optimal systems of $s o(1,3)$ constructed in Theorem 5 and join each one of them with the optimal systems of the abelian algebra $\mathbb{R}^{2}$.

- To get the one-dimensional optimal system of $\mathcal{L}$,

1. either we take $\tilde{\Theta}_{1}$ itself; in this case, we get $\left\{\left\langle V_{8}\right\rangle,\left\langle V_{7}+\beta V_{8}\right\rangle: \beta \in \mathbb{R}\right\}$; 
2. or we add an arbitrary element from $\mathbb{R}^{2}$ to every representative element in $\Theta_{1}$; in this case, we get $\left\{\left\langle V_{4}+Z_{1}\right\rangle,\left\langle V_{5}+Z_{1}\right\rangle,\left\langle V_{3}+\alpha V_{4}+Z_{1}\right\rangle: \alpha \in \mathbb{R}\right\}$.

- To get the two-dimensional optimal system of $\mathcal{L}$,

1. either we add an arbitrary element from $\mathbb{R}^{2}$ to every element in $\Theta_{2}$; in this case, we get $\left\{\left\langle V_{3}+Z_{1}, V_{4}+Z_{2}\right\rangle,\left\langle V_{4}+Z_{1}, V_{5}+Z_{2}\right\rangle,\left\langle V_{5}+Z_{1}, V_{6}+Z_{2}\right\rangle\right\}$,

2. or we add an arbitrary element from $\mathbb{R}^{2}$ to every element in $\Theta_{1}$ and combine the result with an element from $\tilde{\Theta}_{1}$; in this case, we get $\left\{\left\langle V_{4}+Z_{1}, Z_{3}\right\rangle,\left\langle V_{5}+Z_{1}, Z_{3}\right\rangle,\left\langle V_{3}+\alpha V_{4}+Z_{1}, Z_{3}\right\rangle\right.$ : $\alpha \in \mathbb{R}\}$.

3. or take $\tilde{\Theta}_{2}$ itself; in this case, we get $\left\{\left\langle V_{7}, V_{8}\right\rangle\right\}$.

- To get the three-dimensional optimal system of $\mathcal{L}$,

1. either we add an arbitrary element from $\mathbb{R}^{2}$ to every element in $\Theta_{3}$; in this case, we get $\left\{\left\langle V_{4}+Z_{1}, V_{5}+Z_{2}, V_{6}+Z_{3}\right\rangle,\left\langle V_{3}+\alpha V_{4}+Z_{1}, V_{5}+Z_{2}, V_{6}+Z_{3}\right\rangle: \alpha \in \mathbb{R}\right\}$,

2. or we add an arbitrary element from $\mathbb{R}^{2}$ to every element in $\Theta_{2}$ and combine the result with an element from $\tilde{\Theta}_{1}$; in this case, we get $\left\{\left\langle V_{3}+Z_{1}, V_{4}+Z_{2}, Z_{3}\right\rangle,\left\langle V_{4}+Z_{1}, V_{5}+Z_{2}, Z_{3}\right\rangle\right.$, $\left.\left\langle V_{5}+Z_{1}, V_{6}+Z_{2}, Z_{3}\right\rangle\right\}$,

3. or we add an arbitrary element from $\mathbb{R}^{2}$ to every element in $\Theta_{1}$ and combine the result with an element from $\tilde{\Theta}_{2}$; in this case, we get $\left\{\left\langle V_{4}+Z_{1}, V_{7}, V_{8}\right\rangle,\left\langle V_{5}+Z_{1}, V_{7}, V_{8}\right\rangle,\left\langle V_{3}+\alpha V_{4}+\right.\right.$ $\left.\left.Z_{1}, V_{7}, V_{8}\right\rangle: \alpha \in \mathbb{R}\right\}$,

where $Z_{1}=\alpha_{1} V_{7}+\beta_{1} V_{8}, Z_{2}=\alpha_{2} V_{7}+\beta_{2} V_{8}$ are arbitrary elements of $\mathbb{R}^{2}$ and $Z_{3}=V_{7}+\alpha_{3} V_{8}$ or $Z_{3}=V_{8}$ represents a one-dimensional optimal system of $\mathbb{R}^{2}$ and $\alpha_{1}, \alpha_{2}, \alpha_{3}, \beta_{1}, \beta_{2} \in \mathbb{R}$.

Finally, we check that the obtained class is a subalgebra by taking the wedge product of its commutator with each element in the class and make these wedges equal to zero. Therefore, we have the following theorem.

Theorem 6. The optimal systems of solvable subalgebras of $\mathcal{L}$ with the non-zero Lie brackets (22) are as follows:

- The one-dimensional solvable optimal system is $\left\{\left\langle V_{4}+Z_{1}\right\rangle,\left\langle V_{5}+Z_{1}\right\rangle,\left\langle V_{8}\right\rangle,\left\langle V_{3}+\alpha V_{4}+Z_{1}\right\rangle,\left\langle V_{7}+\right.\right.$ $\left.\left.\beta V_{8}\right\rangle: \alpha, \beta \in \mathbb{R}\right\}$.

- The two-dimensional solvable optimal system is

$\left\{\left\langle V_{3}+Z_{1}, V_{4}+Z_{2}\right\rangle,\left\langle V_{4}+Z_{1}, V_{5}\right\rangle,\left\langle V_{5}+Z_{1}, V_{6}+Z_{2}\right\rangle,\left\langle V_{4}+Z_{1}, Z_{3}\right\rangle,\left\langle V_{5}+Z_{1}, Z_{3}\right\rangle,\left\langle V_{7}, V_{8}\right\rangle,\left\langle V_{3}+\right.\right.$ $\left.\left.\alpha V_{4}+Z_{1}, Z_{3}\right\rangle: \alpha \in \mathbb{R}\right\}$.

- The three-dimensional solvable optimal system is $\left\{\left\langle V_{4}+Z_{1}, V_{5}, V_{6}\right\rangle,\left\langle V_{3}+Z_{1}, V_{4}+Z_{2}, Z_{3}\right\rangle\right.$, $\left\langle V_{4}+Z_{1}, V_{5}, Z_{3}\right\rangle,\left\langle V_{5}+Z_{1}, V_{6}+Z_{2}, Z_{3}\right\rangle,\left\langle V_{4}+Z_{1}, V_{7}, V_{8}\right\rangle,\left\langle V_{5}+Z_{1}, V_{7}, V_{8}\right\rangle,\left\langle V_{3}+\alpha V_{4}+Z_{1}\right.$, $\left.\left.V_{5}, V_{6}\right\rangle,\left\langle V_{3}+\alpha V_{4}+Z_{1}, V_{7}, V_{8}\right\rangle: \alpha \in \mathbb{R}\right\}$.

Here, $Z_{1}=\alpha_{1} V_{7}+\beta_{1} V_{8}, Z_{2}=\alpha_{2} V_{7}+\beta_{2} V_{8}$ are arbitrary elements of $\mathbb{R}^{2}$ and $Z_{3}=V_{7}+\alpha_{3} V_{8}$ or $Z_{3}=V_{8}$ represents a one-dimensional optimal system of $\mathbb{R}^{2}$.

\subsubsection{Three-Dimensional Optimal System of Non-Solvable Subalgebras of $\mathcal{L}=s o(1,3) \oplus \mathbb{R}^{2}$}

If $H$ is a three-dimensional non-solvable algebra, then $H$ equals its commutator. As the commutator of $\mathcal{L}$ is so $(1,3)$, all such subalgebras of $\mathcal{L}$ are subalgebras of so $(1,3)$. To find such subalgebras, we follow the algorithm given in Section 2.2.

We need to construct the copies of $s o(3)$ and $s l(2, \mathbb{R})$, if any, by following the algorithm that is given in Section 2.2:

- First, construct the copies of $s o(3)$ : the element $V_{3}$ has the eigenvector $V_{1}+i V_{2}$ corresponding to the eigenvalue $-i$. Therefore, $\tilde{V}_{3}=-V_{3}$ has the same eigenvector with the eigenvalue $i$. Moreover, $\left[V_{1}, V_{2}\right]=-\tilde{V}_{3}$. Therefore, $\left\langle V_{1}, V_{2}, V_{3}\right\rangle$ forms a copy of so(3). 
- The only non-abelian two-dimensional subalgebra in $s o(1,3)$ is $\left\langle V_{4}, V_{5}\right\rangle$ with $\left[V_{4}, V_{5}\right]=c V_{5}$. Moreover, the eigenvector of ad $V_{4}$ corresponding to the eigenvalue $-c$ is $V_{5}-2 c V_{2}$ and $\left[V_{5}-2 c V_{2}, V_{5}\right]=-2 c V_{4}$. Hence, the subalgebra $\left\langle V_{4}, V_{5}, V_{5}-2 c V_{2}\right\rangle$ forms a copy of $s l(2, \mathbb{R})$.

This proves the theorem.

Theorem 7. The three-dimensional non-solvable optimal system is $\left\{\left\langle V_{1}, V_{2}, V_{3}\right\rangle,\left\langle V_{4}, V_{5}, V_{5}-2 c V_{2}\right\rangle\right\}$, where the $V_{i}(i=1, \ldots, 6)$ forms a basis of so $(1,3)$ given in $(21)$.

\section{Joint Invariants and Invariant Solutions Corresponding to Three-Dimensional Optimal Systems of $\mathcal{L}$}

The invariant solutions can be obtained through symmetry reductions carried out by implementing the well-known procedure of utilizing the joint invariants of the subalgebras obtained by three-dimensional optimal system, see, e.g., $[6,11,20]$ for details.

Remark 1 ([10]). Let $\left\langle X_{1}, \ldots, X_{n}\right\rangle$ be a Lie algebra with basis

$$
X_{i}=\xi_{i}^{1} \frac{\partial}{\partial t}+\xi_{i}^{2} \frac{\partial}{\partial r}+\xi_{i}^{3} \frac{\partial}{\partial \theta}+\xi_{i}^{4} \frac{\partial}{\partial \varphi}+\eta_{i} \frac{\partial}{\partial u}, i=1, \ldots, n .
$$

A necessary condition for the existence of an invariant solution under the Lie algebra $\left\langle X_{1}, \ldots, X_{n}\right\rangle$ is the following transversality condition:

$$
\operatorname{rank}\left\{E_{1}\right\}=\operatorname{rank}\left\{E_{2}\right\}
$$

where

$$
E_{1}=\left(\begin{array}{cccc}
\xi_{1}^{1} & \xi_{1}^{2} & \xi_{1}^{3} & \xi_{1}^{4} \\
\vdots & \vdots & \vdots & \vdots \\
\xi_{n}^{1} & \xi_{n}^{2} & \xi_{n}^{3} & \xi_{n}^{4}
\end{array}\right), E_{2}=\left(\begin{array}{ccccc}
\xi_{1}^{1} & \xi_{1}^{2} & \xi_{1}^{3} & \xi_{1}^{4} & \eta_{1} \\
\vdots & \vdots & \vdots & \vdots & \vdots \\
\xi_{n}^{1} & \xi_{n}^{2} & \xi_{n}^{3} & \xi_{n}^{4} & \eta_{n}
\end{array}\right)
$$

Before giving the formal definition of equivalent invariant solutions, let us note the following general fact:

Whenever a transformation group $G$ operates on a set $S$ and $U$ is a subset of $S$ and $H$ is the stabilizer of $U$, then the stabilizer of $a . U, a \in G$ is $a \mathrm{Ha}^{-1}$. We will apply this where the set $S$ is the set of solutions of a differential equation, $U$ is the set of invariant solutions and the group $G$ is the local group whose Lie algebra is the symmetry algebra of the differential equation.

Definition 4. Consider the differential equation admitting the group of transformations $G$. Let $\mathcal{L}$ be the Lie algebra corresponding the group $G$. If $u=\Theta_{1}(x)$ and $u=\Theta_{2}(x)$ are two invariant solutions of the given differential equation under the subalgebras $H_{1}$ and $H_{2}$ of $\mathcal{L}$, respectively, then we call $u=\Theta_{1}(x)$ and $u=\Theta_{2}(x)$ equivalent invariant solutions with respect to the group $G$ if one can find some transformation in $G$ that transforms $u=\Theta_{1}(x)$ to $u=\Theta_{2}(x)$.

Let $H_{1}$ be conjugate to $H_{2}$ with respect to the group of transformations $G$. Define $U$ to be the set of invariant surfaces under $H_{1}$. Then, $H_{1}$ belongs to the stabilizer of $U$ and $H_{2}$ belongs to the stabilizer of $a . U$ for some $a \in G$. The set of invariant surfaces under $H_{2}$ should be of the form a.U.

Therefore, the problem of classifying the invariant solutions is reduced to classifying the corresponding conjugacy classes of subalgebras of the symmetry algebra $\mathcal{L}$ [6].

In this section, we compute the invariant solutions corresponding to three-dimensional subalgebras of $\mathcal{L}$. 


\subsection{Invariant Solutions of the Wave Equation on Einstein Spacetime}

\subsubsection{Solvable Subalgebras of of $\mathcal{L}$}

Example 1. In the case of $\mathcal{L}_{1}=\left\langle V_{1}+Z_{1}, V_{4}+Z_{2}, Z_{3}\right\rangle, Z_{3}=V_{7}+\alpha_{3} V_{8}$. The generators of $\mathcal{L}_{1}$ in Cartesian coordinates are as follows:

$$
\begin{gathered}
V_{1}+Z_{1}=\alpha_{1} \frac{\partial}{\partial t}-c z \frac{\partial}{\partial x}+\sqrt{1-c^{2}\left(x^{2}+y^{2}+z^{2}\right)} \frac{\partial}{\partial y}+c x \frac{\partial}{\partial z}+\beta_{1} u \frac{\partial}{\partial u} \\
V_{4}+Z_{2}=\alpha_{2} \frac{\partial}{\partial t}+c z \frac{\partial}{\partial x}+\sqrt{1-c^{2}\left(x^{2}+y^{2}+z^{2}\right)} \frac{\partial}{\partial y}-c x \frac{\partial}{\partial z}+\beta_{2} u \frac{\partial}{\partial u} \\
Z_{3}=\frac{\partial}{\partial t}+\alpha_{3} u \frac{\partial}{\partial u} .
\end{gathered}
$$

The transversality condition (24) of (25) with rank three is always satisfied. Since the Lie algebra $\mathcal{L}_{1}$ is abelian, one can find the invariant functions, we call them also invariants, of $\mathcal{L}_{1}$ in any order. The invariants of $Z_{3}$ are:

$$
m_{1}=x, m_{2}=y, m_{3}=z, m_{4}=u \mathrm{e}^{-\alpha_{3} t} .
$$

The remaining operators can be given in terms of the variables $m_{i}, i=1, \ldots, 4$ as

$$
\begin{gathered}
V_{1}+Z_{1}=-c m_{3} \frac{\partial}{\partial m_{1}}+\sqrt{1-c^{2}\left(m_{1}^{2}+m_{2}^{2}+m_{3}^{2}\right)} \frac{\partial}{\partial m_{2}}+c m_{1} \frac{\partial}{\partial m_{3}}-m_{4}\left(\alpha_{3} \alpha_{1}-\beta_{1}\right) \frac{\partial}{\partial m_{4}}, \\
V_{4}+Z_{2}=c m_{3} \frac{\partial}{\partial m_{1}}+\sqrt{1-c^{2}\left(m_{1}^{2}+m_{2}^{2}+m_{3}^{2}\right)} \frac{\partial}{\partial m_{2}}-c m_{1} \frac{\partial}{\partial m_{3}}-m_{4}\left(\alpha_{3} \alpha_{2}-\beta_{2}\right) \frac{\partial}{\partial m_{4}} .
\end{gathered}
$$

Next, the invariants of $V_{1}+Z_{1}$ are

$$
\begin{aligned}
& n_{1}=m_{1}^{2}+m_{3}^{2}, n_{2}=\arctan \left(\frac{c m_{2}}{\sqrt{1-c^{2}\left(m_{1}^{2}+m_{2}^{2}+m_{3}^{2}\right)}}\right)-\arctan \left(\frac{m_{3}}{m_{1}}\right), \\
& n_{3}=m_{4} e^{\frac{\left(\alpha_{3} \alpha_{1}-\beta_{1}\right)}{c} \arctan \left(\frac{m_{3}}{m_{1}}\right)} .
\end{aligned}
$$

In terms of the variables $n_{i}, i=1, \ldots, 3$, the remaining operator is

$$
V_{4}+Z_{2}=-2 c \frac{\partial}{\partial n_{2}}-n_{3}\left(\left(\alpha_{1}+\alpha_{2}\right) \alpha_{3}-\beta_{2}-\beta_{1}\right) \frac{\partial}{\partial n_{3}} .
$$

Finally, the invariants of $V_{4}+Z_{2}$ are

$$
n_{1}, n_{3} e^{\frac{\left(\beta_{1}+\beta_{2}-\alpha_{3} \alpha_{1}-\alpha_{3} \alpha_{2}\right)}{2 c} n_{2}}
$$

Writing the invariants (30) in terms of the original variables gives the joint invariants of $\mathcal{L}_{1}$ as

$$
x^{2}+z^{2}, u e^{\left(A_{1} \arctan \left(\frac{c y}{\sqrt{1-c^{2}\left(x^{2}+y^{2}+z^{2}\right)}}\right)+A_{2} \arctan \left(\frac{z}{x}\right)-\alpha_{3} t\right)},
$$

where $A_{1}=\frac{\left(\alpha_{1}+\alpha_{2}\right) \alpha_{3}-\beta_{1}-\beta_{2}}{2 c}, A_{2}=\frac{\left(3 \alpha_{1}+\alpha_{2}\right) \alpha_{3}-3 \beta_{1}-\beta_{2}}{2 c}$.

Note that $A_{1}=A_{2}=0$ when $Z_{1}=Z_{2}=0$. Therefore, for simplicity, let us discuss the invariant solution for this case.

The invariant transformations in this case are:

$$
w=x^{2}+z^{2}, Z(w)=u e^{-\alpha_{3} t} .
$$

Thus, using (32), Equation (8) can be reduced to the ODE:

$$
4 w\left(c^{2} w-1\right) Z^{\prime \prime}+4\left(2 c^{2} w-1\right) Z^{\prime}+\alpha_{3}^{2} Z=0 .
$$


It was found that the transformation

$$
w=\frac{r}{c^{2}}, Z(w)=R(r)
$$

reduces Equation (33) to the hypergeometric differential equation

$$
r(r-1) R^{\prime \prime}+((v+\mu+1) r-\gamma) R^{\prime}+v \mu R=0
$$

with $v=\frac{c-\sqrt{c^{2}-\alpha_{3}^{2}}}{2 c}, \mu=\frac{c+\sqrt{c^{2}-\alpha_{3}^{2}}}{2 c}, \gamma=1$. The solution of (35) is given in terms of the hypergeometric function $F(\nu, \mu ; \gamma ; r)$ as

$$
R(r)=c_{1} F(\mu, v ; v+\mu ; 1-r)+c_{2}(r-1)^{1-v-\mu} F(1-v, 1-\mu ; 2-v-\mu ; 1-r) .
$$

Therefore, the solution of (33) is

$$
Z(w)=R\left(c^{2} w\right)
$$

Thus, the invariant solution of (8) is

$$
u(t, x, z)=e^{\alpha_{3} t}\left(c_{1} F\left(\mu, v ; v+\mu ; 1-c^{2}\left(x^{2}+z^{2}\right)\right)+c_{2}\left(c^{2}\left(x^{2}+z^{2}\right)-1\right)^{1-v-\mu} F\left(1-v, 1-\mu ; 2-v-\mu ; 1-c^{2}\left(x^{2}+z^{2}\right)\right)\right) .
$$

Another interesting special case when $\alpha_{3}=c$, the solution of Equation (35) becomes

$$
R(r)=c_{1} \operatorname{EllipticK}(\sqrt{r})+c_{2} \operatorname{EllipticCK}(\sqrt{r}),
$$

where EllipticK and EllipticCK are respectively the complete and the complementary Elliptic integrals of the first kind.

Thus, the invariant solution of (8) is

$$
u(t, x, z)=e^{c t}\left(c_{1} \text { EllipticK }\left(\sqrt{c^{2}\left(x^{2}+z^{2}\right)}\right)+c_{2} \text { EllipticCK }\left(\sqrt{c^{2}\left(x^{2}+z^{2}\right)}\right)\right) .
$$

\subsubsection{Non-Solvable Subalgebras of of $\mathcal{L}$}

As is well known, all three-dimensional non-solvable subalgebras are simple. As they have no non-trivial ideal, we use the method of reduced row echelon form of operators in any convenient basis. As shown in [21], the operators of the three-dimensional non-solvable subalgebra in the reduced row echelon form always form an abelian algebra. Clearly, the joint invariants of the three-dimensional non-solvable subalgebra are the same as those of this abelian algebra. Using this, we find that the joint invariants for $\mathcal{L}$ as follows:

Example 2. In the case of $\mathcal{L}_{1}=\left\langle V_{1}, V_{2}, V_{3}\right\rangle$, by writing $\mathcal{L}_{1}$ in the reduced row echelon form, the fundamental set of the invariants can be obtained by solving the following system:

$$
\left(\begin{array}{lllll}
0 & 1 & 0 & 0 & 0 \\
0 & 0 & 1 & 0 & 0 \\
0 & 0 & 0 & 1 & 0
\end{array}\right)\left(\begin{array}{c}
I_{t} \\
I_{x} \\
I_{y} \\
I_{z} \\
I_{u}
\end{array}\right)=\left(\begin{array}{l}
0 \\
0 \\
0 \\
0 \\
0
\end{array}\right) .
$$


Clearly, the joint invariants are $t, u$. Therefore, the invariant transformations are:

$$
w=t, Z(w)=u
$$

Thus, using (125), Equation (8) can be reduced to the ODE:

$$
Z^{\prime \prime}=0
$$

which has the solution

$$
Z(w)=c_{1}+c_{2} w
$$

Thus, the invariant solution of (8) is

$$
u(t)=c_{1}+c_{2} t
$$

Example 3. In the case of $\mathcal{L}_{2}=\left\langle V_{4}, V_{5}, V_{6}\right\rangle$, since the reduced row echelon form of the operators of $\mathcal{L}_{2}$ coincides with that in (124), it follows that they have the same solution.

\subsection{Invariant Solutions of the Wave Equation on Anti-Einstein Spacetime}

\subsubsection{Solvable Subalgebras of $\mathcal{L}$}

Example 4. Case $\mathcal{L}_{1}=\left\langle V_{3}+\alpha V_{4}+Z_{1}, V_{5}, V_{6}\right\rangle, \alpha \neq 0$. The generators of $\mathcal{L}_{1}$ in Cartesian coordinates are as follows:

$$
\begin{aligned}
V_{3}+\alpha V_{4}+Z_{1} & =\alpha_{1} \frac{\partial}{\partial t}-y \frac{\partial}{\partial x}+x \frac{\partial}{\partial y}+\alpha \sqrt{1+c^{2}\left(x^{2}+y^{2}+z^{2}\right)} \frac{\partial}{\partial z}, \beta_{1} u \frac{\partial}{\partial u}, \\
V_{5} & =\left(\sqrt{1+c^{2}\left(x^{2}+y^{2}+z^{2}\right)}+c z\right) \frac{\partial}{\partial y}-c y \frac{\partial}{\partial z} \\
V_{6} & =\left(\sqrt{1+c^{2}\left(x^{2}+y^{2}+z^{2}\right)}+c z\right) \frac{\partial}{\partial x}-c x \frac{\partial}{\partial z} .
\end{aligned}
$$

The transversality condition (24) of (46) with rank three is always satisfied. Since the derived Lie algebra generated by $\mathcal{L}_{1}$ is $\left\langle V_{5}, V_{6}\right\rangle$ which is abelian, one can find the invariants of $\mathcal{L}_{1}$ by starting with $V_{5}$ or $V_{6}$. The invariants of $V_{5}$ are:

$$
m_{1}=t, m_{2}=x, m_{3}=\frac{-c z+\sqrt{1+c^{2}\left(x^{2}+y^{2}+z^{2}\right)}}{1+c^{2}\left(x^{2}+y^{2}\right)}, m_{4}=u .
$$

The operator $V_{6}$ can be given in terms of the variables $m_{i}, i=1, \ldots, 4$ as

$$
V_{6}=\frac{1}{m_{3}} \frac{\partial}{\partial m_{2}} .
$$

Next, the invariants of $V_{6}$ are

$$
n_{1}=m_{1}, n_{2}=m_{3}, n_{3}=m_{4} .
$$

In terms of the variables $n_{i}, i=1,2,3$, the remaining operator is

$$
V_{3}+\alpha V_{4}+Z_{1}=\alpha_{1} \frac{\partial}{\partial m_{1}}-\alpha c n_{2} \frac{\partial}{\partial n_{2}}+\beta_{1} n_{3} \frac{\partial}{\partial n_{3}} .
$$

We have to study the following two cases:

- $\quad$ Case 1: If $\alpha_{1} \neq 0$, the invariants of $V_{3}+\alpha V_{4}+Z_{1}$ are

$$
n_{2} \mathrm{e}^{\frac{c \alpha}{\alpha_{1}} n_{1}}, n_{3} \mathrm{e}^{-\frac{\beta_{1}}{\alpha_{1}} n_{1}} .
$$


Writing the invariants (51) in terms of the original variables gives the joint invariants of $\mathcal{L}_{1}$ as

$$
\frac{-c z+\sqrt{1+c^{2}\left(x^{2}+y^{2}+z^{2}\right)}}{1+c^{2}\left(x^{2}+y^{2}\right)} \mathrm{e}^{\frac{\alpha c}{\alpha_{1}} t}, u \mathrm{e}^{-\frac{\beta_{1}}{\alpha_{1}} t} .
$$

Therefore, the invariant transformations are:

$$
w=\frac{-c z+\sqrt{1+c^{2}\left(x^{2}+y^{2}+z^{2}\right)}}{1+c^{2}\left(x^{2}+y^{2}\right)} \mathrm{e}^{\frac{\alpha c}{\alpha_{1}} t}, Z(w)=u e^{-\frac{\beta_{1}}{\alpha_{1}} t} .
$$

Thus, using (52), Equation (12) can be reduced to the ODE:

$$
c\left(\left(\alpha^{2}+\alpha_{1}^{2}\right) c+2 \alpha \beta_{1}\right) w Z^{\prime}+c^{2}\left(\alpha^{2}-\alpha_{1}^{2}\right) w^{2} Z^{\prime \prime}+\beta_{1}^{2} Z=0,
$$

which has the non-trivial solution for the following cases:

1. $\alpha^{2}-\alpha_{1}^{2} \neq 0$ :

$$
Z(w)=c_{1} w^{\frac{-c \alpha_{1}{ }^{2}-\alpha \beta_{1}+\alpha_{1} \sqrt{c^{2} \alpha_{1}{ }^{2}+2 c \alpha \beta_{1}+\beta_{1}{ }^{2}}}{\left(\alpha^{2}-\alpha_{1}{ }^{2}\right) c}}+c_{2} w^{\frac{-c \alpha_{1}{ }^{2}-\alpha \beta_{1}-\alpha_{1} \sqrt{c^{2} \alpha_{1}{ }^{2}+2 c \alpha \beta_{1}+\beta_{1}{ }^{2}}}{\left(\alpha^{2}-\alpha_{1}{ }^{2}\right) c}} .
$$

2. $\alpha^{2}-\alpha_{1}^{2}=0, c \alpha_{1}+\beta_{1} \neq 0$ :

$$
Z(w)=c_{1} w^{-\frac{\beta_{1}^{2}}{2 c \alpha_{1}\left(\alpha_{1} c+\beta_{1}\right)}} .
$$

Thus, the invariant solution of (12) is

$$
u(t, x, y, z)=Z\left(\frac{-c z+\sqrt{1+c^{2}\left(x^{2}+y^{2}+z^{2}\right)}}{1+c^{2}\left(x^{2}+y^{2}\right)} \mathrm{e}^{\frac{\alpha c}{\alpha_{1}} t}\right) e^{\frac{\beta_{1}}{\alpha_{1}} t}
$$

- $\quad$ Case 2: If $\alpha_{1}=0$, the invariants of $V_{3}+\alpha V_{4}+Z_{1}$ are

$$
n_{1}, n_{3} n_{2}^{\frac{\beta_{1}}{\alpha c}} \text {. }
$$

Writing the invariants (57) in terms of the original variables gives the joint invariants of $\mathcal{L}_{1}$ as

$$
t, u\left(\frac{-c z+\sqrt{1+c^{2}\left(x^{2}+y^{2}+z^{2}\right)}}{1+c^{2}\left(x^{2}+y^{2}\right)}\right)^{\frac{\beta_{1}}{\alpha c}} .
$$

Therefore, the invariant transformations are:

$$
w=t, Z(w)=u\left(\frac{-c z+\sqrt{1+c^{2}\left(x^{2}+y^{2}+z^{2}\right)}}{1+c^{2}\left(x^{2}+y^{2}\right)}\right)^{\frac{\beta_{1}}{\alpha c}}
$$

Thus, using (58), Equation (12) can be reduced to the ODE:

$$
\alpha^{2} Z^{\prime \prime}-\beta_{1}\left(\beta_{1}+2 \alpha c\right) Z=0,
$$

which has the solution

$$
Z(w)=c_{1} \mathrm{e}^{\frac{\sqrt{\beta_{1}^{2}+2 \beta_{1} \alpha c}}{\alpha} w}+c_{2} \mathrm{e}^{-\frac{\sqrt{\beta_{1}^{2}+2 \beta_{1} \alpha c}}{\alpha}} w
$$


Thus, the invariant solution of (12) is

$$
u(t, x, y, z)=Z(t)\left(\frac{-c z+\sqrt{1+c^{2}\left(x^{2}+y^{2}+z^{2}\right)}}{1+c^{2}\left(x^{2}+y^{2}\right)}\right)^{-\frac{\beta_{1}}{\alpha c}} .
$$

Example 5. Case $\mathcal{L}_{2}=\left\langle V_{3}+Z_{1}, V_{5}, V_{6}\right\rangle$. The generators of $\mathcal{L}_{2}$ in Cartesian coordinates are as follows:

$$
\begin{gathered}
V_{3}+Z_{1}=\alpha_{1} \frac{\partial}{\partial t}-y \frac{\partial}{\partial x}+x \frac{\partial}{\partial y}+\beta_{1} u \frac{\partial}{\partial u}, \\
V_{5}=\left(\sqrt{1+c^{2}\left(x^{2}+y^{2}+z^{2}\right)}+c z\right) \frac{\partial}{\partial y}-c y \frac{\partial}{\partial z}, \\
V_{6}=\left(\sqrt{1+c^{2}\left(x^{2}+y^{2}+z^{2}\right)}+c z\right) \frac{\partial}{\partial x}-c x \frac{\partial}{\partial z} .
\end{gathered}
$$

The transversality condition (24) of (62) with rank three is satisfied for $\alpha_{1} \neq 0$. Since the derived Lie algebra generated by $\mathcal{L}_{2}$ is $\left\langle V_{5}, V_{6}\right\rangle$ which is abelian, one can find the invariants of $\mathcal{L}_{2}$ by starting with the invariants of $\left\langle V_{5}, V_{6}\right\rangle$ which are given by (49) as

$$
n_{1}=t, n_{2}=\frac{-c z+\sqrt{1+c^{2}\left(x^{2}+y^{2}+z^{2}\right)}}{1+c^{2}\left(x^{2}+y^{2}\right)}, n_{3}=u .
$$

In terms of the variables $n_{i}, i=1,2,3$, the remaining operator is

$$
V_{3}+Z_{1}=\alpha_{1} \frac{\partial}{\partial n_{1}}+\beta_{1} n_{3} \frac{\partial}{\partial n_{3}} .
$$

Finally, the invariants of $V_{3}+Z_{1}$ are

$$
n_{2}, n_{3} \mathrm{e}^{-\frac{\beta_{1}}{\alpha_{1}} n_{1}}
$$

Writing the invariants (65) in terms of the original variables gives the joint invariants of $\mathcal{L}_{2}$ as

$$
\frac{-c z+\sqrt{1+c^{2}\left(x^{2}+y^{2}+z^{2}\right)}}{1+c^{2}\left(x^{2}+y^{2}\right)}, u \mathrm{e}^{-\frac{\beta_{1}}{\alpha_{1}} t} .
$$

Therefore, the invariant transformations are:

$$
w=\frac{-c z+\sqrt{1+c^{2}\left(x^{2}+y^{2}+z^{2}\right)}}{1+c^{2}\left(x^{2}+y^{2}\right)}, Z(w)=u \mathrm{e}^{-\frac{\beta_{1}}{\alpha_{1}} t}
$$

Thus, using (66), Equation (12) can be reduced to the Cauchy-Euler ODE:

$$
w^{2} Z^{\prime \prime}-w Z^{\prime}-\left(\frac{\beta_{1}}{c \alpha_{1}}\right)^{2} Z=0,
$$

which has the solution

$$
Z(w)=c_{1} w^{\frac{c \alpha_{1}+\sqrt{c^{2} \alpha_{1}^{2}+\beta_{1}^{2}}}{c \alpha_{1}}}+c_{2} w^{\frac{c \alpha_{1}-\sqrt{c^{2} \alpha_{1}^{2}+\beta_{1}^{2}}}{c \alpha_{1}}} .
$$

Thus, the invariant solution of (12) is

$$
u(t, x, y, z)=Z\left(\frac{-c z+\sqrt{1+c^{2}\left(x^{2}+y^{2}+z^{2}\right)}}{1+c^{2}\left(x^{2}+y^{2}\right)}\right) \mathrm{e}^{\frac{\beta_{1}}{\alpha_{1}} t}
$$


Example 6. Case $\mathcal{L}_{3}=\left\langle V_{4}+Z_{1}, V_{5}, V_{6}\right\rangle$. The generators of $\mathcal{L}_{3}$ in Cartesian coordinates are as follows:

$$
\begin{gathered}
V_{4}+Z_{1}=\alpha_{1} \frac{\partial}{\partial t}+\sqrt{1+c^{2}\left(x^{2}+y^{2}+z^{2}\right)} \frac{\partial}{\partial z}+\beta_{1} u \frac{\partial}{\partial u}, \\
V_{5}=\left(\sqrt{1+c^{2}\left(x^{2}+y^{2}+z^{2}\right)}+c z\right) \frac{\partial}{\partial y}-c y \frac{\partial}{\partial z}, \\
V_{6}=\left(\sqrt{1+c^{2}\left(x^{2}+y^{2}+z^{2}\right)}+c z\right) \frac{\partial}{\partial x}-c x \frac{\partial}{\partial z} .
\end{gathered}
$$

The transversality condition (24) of (70) with rank three is always satisfied. Since the derived Lie algebra generated by $\mathcal{L}_{3}$ is $\left\langle V_{5}, V_{6}\right\rangle$ which is abelian, one can find the invariants of $\mathcal{L}_{3}$ by starting with the invariants of $\left\langle V_{5}, V_{6}\right\rangle$ which are given by (49) as

$$
n_{1}=t, n_{2}=\frac{-c z+\sqrt{1+c^{2}\left(x^{2}+y^{2}+z^{2}\right)}}{1+c^{2}\left(x^{2}+y^{2}\right)}, n_{3}=u .
$$

In terms of the variables $n_{i}, i=1,2,3$, the remaining operator is

$$
V_{4}+Z_{1}=\alpha_{1} \frac{\partial}{\partial n_{1}}-c n_{2} \frac{\partial}{\partial n_{2}}+\beta_{1} n_{3} \frac{\partial}{\partial n_{3}} .
$$

We have to consider the following two cases:

- $\quad$ Case 1: If $\alpha_{1} \neq 0$, the invariants of $V_{4}+Z_{1}$ are

$$
n_{2} \mathrm{e}^{\frac{c}{\alpha_{1}} n_{1}}, n_{3} \mathrm{e}^{-\frac{\beta_{1}}{\alpha_{1}} n_{1}}
$$

Writing the invariants (73) in terms of the original variables gives the joint invariants of $\mathcal{L}_{3}$ as

$$
\frac{-c z+\sqrt{1+c^{2}\left(x^{2}+y^{2}+z^{2}\right)}}{1+c^{2}\left(x^{2}+y^{2}\right)} \mathrm{e}^{\frac{c t}{\alpha_{1}}}, u \mathrm{e}^{-\frac{\beta_{1}}{\alpha_{1}} t} .
$$

Therefore, the invariant transformations are:

$$
w=\frac{-c z+\sqrt{1+c^{2}\left(x^{2}+y^{2}+z^{2}\right)}}{1+c^{2}\left(x^{2}+y^{2}\right)} \mathrm{e}^{\frac{c t}{\alpha_{1}}}, Z(w)=u e^{-\frac{\beta_{1}}{\alpha_{1}} t} .
$$

Thus, using (75), Equation (12) can be reduced to the ODE:

$$
c^{2}\left(\alpha_{1}^{2}-1\right) w^{2} Z^{\prime \prime}-\left(\left(1+\alpha_{1}^{2}\right) c+2 \beta_{1}\right) c w Z^{\prime}-\beta_{1}^{2} Z=0,
$$

which has the non-trivial solution for the following cases:

1. $\alpha_{1}^{2}-1 \neq 0$ :

$$
Z(w)=c_{1} w^{\frac{c \alpha_{1}^{2}+\beta_{1}+\alpha_{1} \sqrt{c^{2} \alpha_{1}^{2}+2 c \beta_{1}+\beta_{1}^{2}}}{c\left(\alpha_{1}^{2}-1\right)}}+c_{2} w^{\frac{c \alpha_{1}^{2}+\beta_{1}-\alpha_{1} \sqrt{c^{2} \alpha_{1}^{2}+2 c \beta_{1}+\beta_{1}^{2}}}{c\left(\alpha_{1}^{2}-1\right)}}
$$

2. $\alpha_{1}^{2}-1=0, c+\beta_{1} \neq 0$ :

$$
Z(w)=c_{1} w^{-\frac{\beta_{1}^{2}}{2 c\left(c+\beta_{1}\right)}} .
$$


Thus, the invariant solution of (12) is

$$
u(t, x, y, z)=Z\left(\frac{-c z+\sqrt{1+c^{2}\left(x^{2}+y^{2}+z^{2}\right)}}{1+c^{2}\left(x^{2}+y^{2}\right)} \mathrm{e}^{\frac{c t}{\alpha_{1}}}\right) e^{\frac{\beta_{1}}{\alpha_{1}} t} .
$$

- $\quad$ Case 2: If $\alpha_{1}=0$, the invariants of $V_{4}+Z_{1}$ are

$$
n_{1}, n_{3} n_{2}^{\frac{\beta_{1}}{c}} \text {. }
$$

Writing the invariants (80) in terms of the original variables gives the joint invariants of $\mathcal{L}_{3}$ as

$$
t, u\left(\frac{-c z+\sqrt{1+c^{2}\left(x^{2}+y^{2}+z^{2}\right)}}{1+c^{2}\left(x^{2}+y^{2}\right)}\right)^{\frac{\beta_{1}}{c}} .
$$

Therefore, the invariant transformations are:

$$
w=t, Z(w)=u\left(\frac{-c z+\sqrt{1+c^{2}\left(x^{2}+y^{2}+z^{2}\right)}}{1+c^{2}\left(x^{2}+y^{2}\right)}\right)^{\frac{\beta_{1}}{c}} .
$$

Thus, using (82), Equation (12) can be reduced to the Cauchy-Euler ODE:

$$
Z^{\prime \prime}-\left(2 \beta_{1} c+\beta_{1}^{2}\right) Z=0
$$

which has the solution

$$
Z(w)=c_{1} \mathrm{e}^{\sqrt{\beta_{1}^{2}+2 c \beta_{1}} w}+c_{2} \mathrm{e}^{-\sqrt{\beta_{1}^{2}+2 c \beta_{1}} w}
$$

Thus, the invariant solution of (12) is

$$
u(t, x, y, z)=Z(t)\left(\frac{-c z+\sqrt{1+c^{2}\left(x^{2}+y^{2}+z^{2}\right)}}{1+c^{2}\left(x^{2}+y^{2}\right)}\right)^{-\frac{\beta_{1}}{c}}
$$

Example 7. Case $\mathcal{L}_{4}=\left\langle V_{3}+Z_{1}, V_{4}+Z_{2}, Z_{3}\right\rangle, Z_{3}=V_{7}+\alpha_{3} V_{8}$. The generators of $\mathcal{L}_{4}$ in Cartesian coordinates are as follows:

$$
\begin{gathered}
V_{3}+Z_{1}=\alpha_{1} \frac{\partial}{\partial t}-y \frac{\partial}{\partial x}+x \frac{\partial}{\partial y}+\beta_{1} u \frac{\partial}{\partial u} \\
V_{4}+Z_{2}=\alpha_{2} \frac{\partial}{\partial t}+\sqrt{1+c^{2}\left(x^{2}+y^{2}+z^{2}\right)} \frac{\partial}{\partial z}+\beta_{2} u \frac{\partial}{\partial u}, \\
Z_{3}=\frac{\partial}{\partial t}+\alpha_{3} u \frac{\partial}{\partial u} .
\end{gathered}
$$

The transversality condition (24) of (86) with rank three is always satisfied. Since the Lie algebra generated by $\mathcal{L}_{4}$ is abelian, one can find the invariants of $\mathcal{L}_{4}$ in any order. The invariants of $Z_{3}$ are:

$$
m_{1}=x, m_{2}=y, m_{3}=z, m_{4}=u \mathrm{e}^{-\alpha_{3} t} .
$$

The operators can be given in terms of the variables $m_{i}, i=1, \ldots, 4$ as

$$
\begin{gathered}
V_{3}+Z_{1}=-m_{2} \frac{\partial}{\partial m_{1}}+m_{1} \frac{\partial}{\partial m_{2}}+\left(\beta_{1}-\alpha_{3} \alpha_{1}\right) m_{4} \frac{\partial}{\partial m_{4}}, \\
V_{4}+Z_{2}=\sqrt{1+c^{2}\left(m_{1}^{2}+m_{2}^{2}+m_{3}^{2}\right)} \frac{\partial}{\partial m_{3}}+\left(\beta_{2}-\alpha_{2} \alpha_{3}\right) m_{4} \frac{\partial}{\partial m_{4}} .
\end{gathered}
$$


Next, the invariants of $V_{3}+Z_{1}$ are

$$
n_{1}=m_{1}{ }^{2}+m_{2}{ }^{2}, n_{2}=m_{3}, n_{3}=m_{4} \mathrm{e}^{\left(\beta_{1}-\alpha_{3} \alpha_{1}\right) \arctan \left(\frac{m_{1}}{m_{2}}\right)} .
$$

In terms of the variables $n_{i}, i=1,2,3$, the remaining operator is

$$
V_{4}+Z_{2}=\sqrt{1+c^{2}\left(n_{1}+n_{2}^{2}\right)} \frac{\partial}{\partial n_{1}}+\left(\beta_{2}-\alpha_{2} \alpha_{3}\right) n_{3} \frac{\partial}{\partial n_{3}} .
$$

Finally, the invariants of $V_{4}+Z_{2}$ are

$$
n_{1}, n_{3}\left(c n_{2}+\sqrt{1+c^{2}\left(n_{1}+n_{2}^{2}\right)}\right)^{\frac{\alpha_{2} \alpha_{3}-\beta_{2}}{c}} .
$$

Writing the invariants (91) in terms of the original variables gives the joint invariants of $\mathcal{L}_{4}$ as

$$
x^{2}+y^{2}, u \mathrm{e}^{-\alpha_{3} t} \mathrm{e}^{A_{1} \arctan \left(\frac{x}{y}\right)}\left(c z+\sqrt{1+c^{2}\left(x^{2}+y^{2}+z^{2}\right)}\right)^{A_{2}},
$$

where $A_{1}=\left(\beta_{1}-\alpha_{3} \alpha_{1}\right), A_{2}=\frac{\alpha_{2} \alpha_{3}-\beta_{2}}{c}$.

Therefore, the invariant transformations are:

$$
w=x^{2}+y^{2}, Z(w)=u \mathrm{e}^{-\alpha_{3} t} \mathrm{e}^{A_{1} \arctan \left(\frac{x}{y}\right)}\left(c z+\sqrt{1+c^{2}\left(x^{2}+y^{2}+z^{2}\right)}\right)^{A_{2}} .
$$

Thus, using (93), Equation (12) can be reduced to the ODE:

$$
4\left(w^{2}+c^{2} w^{3}\right) Z^{\prime \prime}+4\left(w-c^{2}\left(A_{2}-2\right) w^{2}\right) Z^{\prime}+\left(\left(\left(A_{2}^{2}-2 A_{2}\right) c^{2}-\alpha^{2}\right) w+A_{1}^{2}\right) Z=0,
$$

which can be transformed using the transformation $w=-\frac{r}{c^{2}}$.

$Z(w)=r^{\frac{1}{2}} i A_{1} R(r)$ to the hypergeometric differential equation

$$
r(r-1) R^{\prime \prime}+((\nu+\mu+1) r-\gamma) R^{\prime}+v \mu R=0
$$

with $v=\frac{\left(1+i A_{1}-A_{2}\right) c+\sqrt{c^{2}+\alpha_{3}^{2}}}{2 c}, \mu=\frac{\left(1+i A_{1}-A_{2}\right) c-\sqrt{c^{2}+\alpha_{3}^{2}}}{2 c}, \gamma=1+i A_{1}$. The solution of (95) is given in terms of the hypergeometric function $F(\nu, \mu ; \gamma ; r)$ as

$$
R(r)=c_{1} F(\nu, \mu ; \gamma ; r)+c_{2} r^{1-\gamma} F(v-\gamma+1, \mu-\gamma+1 ; 2-\gamma ; r) .
$$

Therefore, the solution of (94) is

$$
Z(w)=\left(-c^{2} w\right)^{\frac{1}{2}(\gamma-1)} R\left(-c^{2} w\right) .
$$

Thus, the invariant solution of (12) is

$$
u(t, x, y, z)=Z\left(x^{2}+y^{2}\right) \mathrm{e}^{\alpha_{3} t} \mathrm{e}^{i(\gamma-1) \arctan \left(\frac{x}{y}\right)}\left(c z+\sqrt{1+c^{2}\left(x^{2}+y^{2}+z^{2}\right)}\right)^{3 v-\gamma-\mu},
$$

where $\alpha_{3}^{2}=c^{2}(v-\mu)^{2}-c^{2}$. 
Example 8. Case $\mathcal{L}_{5}=\left\langle V_{4}+Z_{1}, V_{5}, Z_{3}\right\rangle, Z_{3}=V_{7}+\alpha_{3} V_{8}$. The generators of $\mathcal{L}_{5}$ in Cartesian coordinates are as follows:

$$
\begin{gathered}
V_{4}+Z_{1}=\alpha_{1} \frac{\partial}{\partial t}+\sqrt{1+c^{2}\left(x^{2}+y^{2}+z^{2}\right)} \frac{\partial}{\partial z}+\beta_{1} u \frac{\partial}{\partial u}, \\
V_{5}=\left(\sqrt{1+c^{2}\left(x^{2}+y^{2}+z^{2}\right)}+c z\right) \frac{\partial}{\partial y}-c y \frac{\partial}{\partial z}, \\
Z_{3}=\frac{\partial}{\partial t}+\alpha_{3} u \frac{\partial}{\partial u} .
\end{gathered}
$$

The transversality condition (24) of (99) with rank three is always satisfied. Since the derived Lie algebra generated by $\mathcal{L}_{5}$ is $\left\langle V_{5}\right\rangle$, one can find the invariants of $\mathcal{L}_{5}$ by starting with $V_{5}$. The invariants of $V_{5}$ are:

$$
m_{1}=t, m_{2}=x, m_{3}=u, m_{4}=\frac{-c z+\sqrt{1+c^{2}\left(x^{2}+y^{2}+z^{2}\right)}}{1+c^{2}\left(x^{2}+y^{2}\right)} .
$$

The operators can be given in terms of the variables $m_{i}, i=1, \ldots, 4$ as

$$
\begin{gathered}
V_{4}+Z_{1}=\alpha_{1} \frac{\partial}{\partial m_{1}}+\beta_{1} m_{3} \frac{\partial}{\partial m_{3}}-c m_{4} \frac{\partial}{\partial m_{4}}, \\
Z_{3}=\frac{\partial}{\partial t}+\alpha_{3} m_{3} \frac{\partial}{\partial m_{3}} .
\end{gathered}
$$

Next, the invariants of $V_{4}+Z_{1}$ are

$$
n_{1}=m_{2}, n_{2}=m_{3} \mathrm{e}^{-\frac{\beta_{1}}{\alpha_{1}} m_{1}}, n_{3}=m_{4} \mathrm{e}^{\frac{c}{\alpha_{1}} m_{1}} .
$$

In terms of the variables $n_{i}, i=1,2,3$, the remaining operator is

$$
Z_{3}=\frac{\left(\alpha_{3} \alpha_{1}-\beta_{1}\right)}{\alpha_{1}} n_{2} \frac{\partial}{\partial n_{2}}+\frac{c}{\alpha_{1}} n_{3} \frac{\partial}{\partial n_{3}} .
$$

Finally, the invariants of $Z_{3}$ are

$$
n_{1}, n_{2} n_{3}^{\frac{\beta_{1}-\alpha_{3} \alpha_{1}}{c}}
$$

Writing the invariants (104) in terms of the original variables gives the joint invariants of $\mathcal{L}_{5}$ as

$$
x, u e^{-\alpha_{3} t}\left(\frac{1+c^{2}\left(x^{2}+y^{2}\right)}{-c z+\sqrt{1+c^{2}\left(x^{2}+y^{2}+z^{2}\right)}}\right)^{A_{1}},
$$

where $A_{1}=\frac{\beta_{1}-\alpha_{1} \alpha_{3}}{c}$.

Therefore, the invariant transformations are:

$$
w=x, Z(w)=u e^{-\alpha_{3} t}\left(\frac{1+c^{2}\left(x^{2}+y^{2}\right)}{-c z+\sqrt{1+c^{2}\left(x^{2}+y^{2}+z^{2}\right)}}\right)^{A_{1}} .
$$

Thus, using (106), Equation (12) can be reduced to the ODE:

$$
\left(c^{2} w^{2}+1\right) Z^{\prime \prime}-c^{2}\left(2 A_{1}-3\right) w Z^{\prime}+\left(c^{2}\left(A_{1}^{2}-2 A_{1}\right)-\alpha_{3}^{2}\right) Z=0 .
$$

It was found using Maple software (Maple 13.0, Waterloo Maple Inc., Waterloo, ON, Canada) that the transformation

$$
w=\frac{r}{i c}, Z(w)=\left(r^{2}-1\right)^{\frac{1}{2} A_{1}-\frac{1}{4}} R(r)
$$

reduces Equation (107) to the associated Legendre equation

$$
\left(1-r^{2}\right) R^{\prime \prime}-2 r R^{\prime}+\left(v(v+1)-\frac{\mu^{2}}{1-r^{2}}\right) R=0
$$


with $v=\frac{2 \sqrt{c^{2}+\alpha_{3}^{2}}-c}{2 c}, \mu=A_{1}-\frac{1}{2}$. Therefore, the solution of (107) is

$$
Z(w)=\left(-c^{2} w^{2}-1\right)^{\frac{\mu}{2}}\left(c_{1} \mathrm{P}_{v}^{\mu}(i c w)+c_{2} \mathbf{Q}_{v}^{\mu}(i c w)\right)
$$

where $\mathrm{P}_{v}^{\mu}$ and $\mathrm{Q}_{v}^{\mu}$ are the associated Legendre functions of the first and second kinds respectively. Thus, the invariant solution of (12) is

$$
u(t, x, y, z)=Z(x) e^{\alpha_{3} t}\left(\frac{-c z+\sqrt{1+c^{2}\left(x^{2}+y^{2}+z^{2}\right)}}{1+c^{2}\left(x^{2}+y^{2}\right)}\right)^{\mu+\frac{1}{2}},
$$

where $\alpha_{3}^{2}=c^{2}\left(v+\frac{1}{2}\right)^{2}-c^{2}$.

Example 9. Case $\mathcal{L}_{6}=\left\langle V_{5}+Z_{1}, V_{6}+Z_{2}, Z_{3}\right\rangle, Z_{3}=V_{7}+\alpha_{3} V_{8}$. The generators of $\mathcal{L}_{6}$ in Cartesian coordinates are as follows:

$$
\begin{gathered}
V_{5}+Z_{1}=\alpha_{1} \frac{\partial}{\partial t}+\left(\sqrt{1+c^{2}\left(x^{2}+y^{2}+z^{2}\right)}+c z\right) \frac{\partial}{\partial y}-c y \frac{\partial}{\partial z}+\beta_{1} u \frac{\partial}{\partial u}, \\
V_{6}+Z_{2}=\alpha_{2} \frac{\partial}{\partial t}+\left(\sqrt{1+c^{2}\left(x^{2}+y^{2}+z^{2}\right)}+c z\right) \frac{\partial}{\partial x}-c x \frac{\partial}{\partial z}+\beta_{2} u \frac{\partial}{\partial u}, \\
Z_{3}=\frac{\partial}{\partial t}+\alpha_{3} u \frac{\partial}{\partial u} .
\end{gathered}
$$

The transversality condition (24) of (112) with rank three is always satisfied. Since the Lie algebra generated by $\mathcal{L}_{6}$ is abelian, one can find the invariants of $\mathcal{L}_{6}$ in any order. The invariants of $Z_{3}$ are:

$$
m_{1}=x, m_{2}=y, m_{3}=z, m_{4}=u \mathrm{e}^{-\alpha_{3} t} .
$$

The operators can be given in terms of the variables $m_{i}, i=1, \ldots, 4$ as

$$
\begin{gathered}
V_{5}+Z_{1}=\left(\sqrt{1+c^{2}\left(m_{1}^{2}+m_{2}^{2}+m_{3}^{2}\right)}+c m_{3}\right) \frac{\partial}{\partial m_{2}}-c m_{2} \frac{\partial}{\partial m_{3}}+\left(\beta_{1}-\alpha_{3} \alpha_{1}\right) m_{4} \frac{\partial}{\partial m_{4}}, \\
V_{6}+Z_{2}=\left(\sqrt{1+c^{2}\left(m_{1}^{2}+m_{2}^{2}+m_{3}^{2}\right)}+c m_{3}\right) \frac{\partial}{\partial m_{1}}-c m_{1} \frac{\partial}{\partial m_{3}}+\left(\beta_{2}-\alpha_{2} \alpha_{3}\right) m_{4} \frac{\partial}{\partial m_{4}} .
\end{gathered}
$$

Next, the invariants of $V_{5}+Z_{1}$ are

$$
\begin{aligned}
& n_{1}=m_{1} \\
& n_{2}=\frac{-c m_{3}+\sqrt{1+c^{2}\left(m_{1}^{2}+m_{2}{ }^{2}+m_{3}^{2}\right)}}{1+c^{2}\left(m_{1}^{2}+m_{2}^{2}\right)}, \\
& n_{3}=m_{4} \mathrm{e}^{\frac{\left(\alpha_{3} \alpha_{1}-\beta_{1}\right) m_{2}\left(-c m_{3}+\sqrt{1+c^{2}\left(m_{1}{ }^{2}+m_{2}{ }^{2}+m_{3}{ }^{2}\right)}\right)}{1+c^{2}\left(m_{1}{ }^{2}+m_{2}{ }^{2}\right)}} .
\end{aligned}
$$

In terms of the variables $n_{i}, i=1,2,3$, the remaining operator is

$$
V_{6}+Z_{2}=\frac{1}{n_{2}} \frac{\partial}{\partial n_{1}}+\left(\beta_{2}-\alpha_{2} \alpha_{3}\right) n_{3} \frac{\partial}{\partial n_{3}} .
$$

Finally, the invariants of $V_{6}+Z_{2}$ are

$$
n_{2}, n_{3} \mathrm{e}^{\left(\alpha_{2} \alpha_{3}-\beta_{2}\right) n_{1} n_{2}}
$$

Writing the invariants (117) in terms of the original variables gives the joint invariants of $\mathcal{L}_{6}$ as

$$
\frac{-c z+\sqrt{1+c^{2}\left(x^{2}+y^{2}+z^{2}\right)}}{1+c^{2}\left(x^{2}+y^{2}\right)}, u e^{-\alpha t} e^{\left(A_{1} x+A_{2} y\right)\left(\frac{-c z+\sqrt{1+c^{2}\left(x^{2}+y^{2}+z^{2}\right)}}{1+c^{2}\left(x^{2}+y^{2}\right)}\right)},
$$

where $A_{1}=\alpha_{2} \alpha_{3}-\beta_{2}, A_{2}=\alpha_{1} \alpha_{3}-\beta_{1}$. 
Therefore, the invariant transformations are:

$$
w=\frac{-c z+\sqrt{1+c^{2}\left(x^{2}+y^{2}+z^{2}\right)}}{1+c^{2}\left(x^{2}+y^{2}\right)}, Z(w)=u e^{-\alpha t} e^{\left(A_{1} x+A_{2} y\right)\left(\frac{-c z+\sqrt{1+c^{2}\left(x^{2}+y^{2}+z^{2}\right)}}{1+c^{2}\left(x^{2}+y^{2}\right)}\right)} .
$$

Thus, using (119), Equation (12) can be reduced to the ODE:

$$
c^{2} w^{2} Z^{\prime \prime}-c^{2} w Z^{\prime}+\left(\left(A_{1}^{2}+A_{2}^{2}\right) w^{2}-\alpha_{3}^{2}\right) Z=0,
$$

which can be transformed using the transformation $w=r, Z(w)=r R(r)$ to the parametric Bessel equation:

$$
r^{2} R^{\prime \prime}+r R^{\prime}+\left(\alpha^{2} r^{2}-v^{2}\right) R=0
$$

with $\alpha=\frac{\sqrt{A_{1}^{2}+A_{2}^{2}}}{c}, v=\frac{\sqrt{c^{2}+\alpha^{2}}}{c}$.

Therefore, the solution of (120) is

$$
\mathrm{Z}(w)=c_{1} w \mathrm{~J}_{v}(\alpha w)+c_{2} w \mathrm{Y}_{v}(\alpha w),
$$

where $\mathrm{J}_{v}$ and $\mathrm{Y}_{v}$ are the Bessel functions of the first and second kind, respectively. Thus, the invariant solution of (12) is

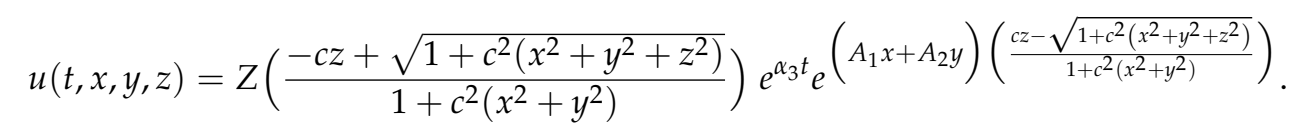

\subsubsection{Non-Solvable Subalgebras of $\mathcal{L}$}

Example 10. Case $\mathcal{L}_{1}=\left\langle V_{4}, V_{5}, V_{5}-2 c V_{2}\right\rangle$. By writing $\mathcal{L}_{1}$ in the reduced row echelon form, the fundamental set of the invariants can be obtained by solving the following system:

$$
\left(\begin{array}{lllll}
0 & 1 & 0 & 0 & 0 \\
0 & 0 & 1 & 0 & 0 \\
0 & 0 & 0 & 1 & 0
\end{array}\right)\left(\begin{array}{c}
I_{t} \\
I_{x} \\
I_{y} \\
I_{z} \\
I_{u}
\end{array}\right)=\left(\begin{array}{l}
0 \\
0 \\
0 \\
0 \\
0
\end{array}\right)
$$

Clearly, the joint invariants are $t, u$. Therefore, the invariant transformations are:

$$
w=t, Z(w)=u
$$

Thus, using (125), Equation (12) can be reduced to the ODE:

$$
Z^{\prime \prime}=0,
$$

which has the solution

$$
Z(w)=c_{1}+c_{2} w .
$$

Thus, the invariant solution of (12) is

$$
u(t)=c_{1}+c_{2} t
$$




\section{Concluding Remarks and Future Research}

Improved algorithms of the expansion method introduced by Ovsiannikov [6] are introduced to construct the optimal systems of dimension of at most three of non-solvable Lie algebra. The algorithms are then applied to determine the Lie algebra structure and optimal systems of the symmetries of the wave equation on static spherically symmetric spacetimes admitting $G_{7}$ as an isometry algebra, while joint invariants and invariant solutions corresponding to three-dimensional optimal systems are also found. The energy density $e(u)=\frac{1}{2} g^{i j} u_{i} u_{j}$ and the corresponding energy of the solutions can be investigated for physical significance of the wave functions obtained in the examples.

It would be of interest to complete and extend this study by applying the algorithms in this paper to equations of physical interest on all static and non-static spherically symmetric spacetimes and to find the corresponding invariant solutions.

Author Contributions: Conceptualization, H.A., A.Y.A.-D. and M.T.M.; Formal analysis, A.Y.A.-D. and M.T.M.; Investigation, H.A., K.A., A.Y.A.-D. and M.T.M.; Methodology, K.A. and A.Y.A.-D.; Supervision, A.Y.A.-D. and M.T.M.; Writing—original draft, H.A. and A.Y.A.-D.; Writing—review \& editing, K.A., A.Y.A.-D. and M.T.M.

Acknowledgments: The publication of this article was funded by the Qatar National Library. The authors are thankful to Qatar University and King Fahd university of petroleum and minerals for their continuous support and excellent research facilities.

Conflicts of Interest: The authors declare no conflict of interest.

\section{References}

1. Azad, H.; Ziad, M. Spherically symmetric manifolds which admit five isometries. J. Math. Phys. 1995, 36, 1908-1911. [CrossRef]

2. Bokhari, A.H.; Qadir, A. Symmetries of static, spherically symmetric space-times. J. Math. Phys. 1987, 28, 1019-1022. [CrossRef]

3. Qadir, A.; Ziad, M. The classification of spherically symmetric space-times. Il Nuovo Cimento B (1971-1996) 1995, 110, 317-334. [CrossRef]

4. Azad, H.; Al-Dweik, A.Y.; Ghanam, R.; Mustafa, M.T. Symmetry analysis of wave equation on static spherically symmetric spacetimes with higher symmetries. J. Math. Phys. 2013, 54, 063509. [CrossRef]

5. Ibragimov, N.H. Transformation Groups Applied to Mathematical Physics; Springer Science \& Business Media: New York, NY, USA, 2001; Volume 3.

6. Ovsiannikov, L.V. Group Analysis of Differential Equations; Academic Press: New York, NY, USA, 1982.

7. Ovsiannikov, L.V. Group Properties of Differential Equations; Siberian Branch, USSR Academy of Sciences: Novosibirsk, Russia, 1962.

8. Ibragimov, N.H. Optimal Systems of Subgroups and Classification of Invariant Solutions of Equations for Planar Non-stationary Gas Flows. Master of Science Thesis in Mathematics, Institute of Hydrodynamics, USSR Academy Science, Novosibirsk State University, Novosibirsk, Rrussia, 1965.

9. Ibragimov, N.H. Selected Works; ALGA Publication: Karlskrona, Sweden, 2006; Volume II.

10. Olver, P.J. Applications of Lie Groups to Differential Equations; Springer Science and Business Media: New York, NY, USA, 2000; Volume 107.

11. Hydon, P.E. Symmetry Methods for Differential Equations: A Beginner's Guide; Cambridge University Press: Cambridge, UK, 2000; Volume 22.

12. Azad, H.; Biswas, I.; Chatterjee, P. On the maximal solvable subgroups of semisimple algebraic groups. J. Lie Theory Vol. 2012, 22, 1169-1179.

13. Ali, S.; Azad, H.; Biswas, I.; Ghanam, R.; Mustafa, M.T. Embedding algorithms and applications to differential equations. J. Symb. Comput. 2018, 86, 166-188. [CrossRef]

14. Hilgert, J.; Neeb, K.H. Structure and Geometry of Lie Groups; Springer: New York, NY, USA, 2011.

15. De Graaf, W.A. Lie Algebras: Theory and Algorithms, North-Holland Mathematical Library; Elsevier Science: Amsterdam, The Netherlands, 2000.

16. Knapp, A.W. Lie Groups beyond an Introduction, 2nd ed.; Progress in Mathematics; Birkhäuser Boston, Inc.: Boston, MA, USA, 2002.

17. Azad, H.; Biswas, I. A note on real algebraic groups. Forum Math. 2016, 28, 539-543. [CrossRef] 
18. Yuri, B.; Freire, I.L. Special conformal groups of a Riemannian manifold and Lie point symmetries of the nonlinear Poisson equation. J. Differ. Equat. 2010, 249, 872-913.

19. Libor, S.; Winternitz, P. Classification and Identification of Lie Algebras; American Mathematical Society: Provence, RI, USA, 2014; Volume 33.

20. Bluman, G.; Stephen, C.A. Symmetry and Integration Methods for Differential Equations; Springer Science, Business Media: New York, NY, USA, 2002; Volume 154.

21. Azad, H.; Biswas, I.; Ghanam, R.; Mustafa, M.T. On computing joint invariants of vector fields. J. Geom. Phys. 2015, 97, 69-76. [CrossRef]

(C) 2018 by the authors. Licensee MDPI, Basel, Switzerland. This article is an open access article distributed under the terms and conditions of the Creative Commons Attribution (CC BY) license (http://creativecommons.org/licenses/by/4.0/). 